\title{
USO DE ESTIMADOS DE DENSIDAD KERNEL EN LA INVESTIGACIÓN DE GRUPOS CERÁMICOS DEL PERIODO FORMATIVO PROVENIENTES DE CHAVÍN DE HUÁNTAR
}

\author{
Christian Mesía Montenegro \\ Museo de ARTE PreColombino CASA DEL Alabado \\ cmesia@alabado.org
}

\section{Resumen}

En este artículo se analiza un grupo de 3020 fragmentos de cerámica diagnóstica recuperadas en cinco unidades espaciales identificadas en Chavín de Huántar, con la finalidad de identificar patrones de asociación entre categorías de diámetro y grosor por tipo de vasija y diferencias de patrones entre las cinco unidades espaciales. Se han utilizado Estimados de Densidad Kernel (EDK) como metodología central en el presente artículo.

Palabras clave: Estimados de densidad kernel, histogramas, modos, cerámica, bivariado, univariado, Chavín, Formativo, Perú.

\section{ABSTRACT}

In this paper I analyze a sub set of 3020 diagnostic ceramic sherds recovered in five spatial units identified in Chavín de Huántar, Peru, in order to find patterns of association between the categories of diameter and thickness per type of ceramic vessel and to identify possible differences of those patterning's among spatial units. I have used Kernel Density Estimates (KDE) for the purpose of identify modalities in diameters and thickness per type in each spatial unit.

KEYwORDs: Kernel density estimations, histograms, modes, ceramics, bivariate, univariate, Chavin, Formative, Peru. 


\section{INTRODUCCIÓN}

En este artículo se presentan ejemplos del uso de rutinas estadísticas de Estimados de Densidad Kernel (EDK) para análisis y presentación de datos, los cuales se distancian del uso tradicional de histogramas. Los histogramas han sido extensamente utilizados como instrumentos de presentación de datos e incluso como herramientas de análisis espacial a pesar de la existencia de otras alternativas (Baxter y Beardah 1996; Baxter et al. 1997; Orton 1988). Se ha argumentado que los histogramas son ineficientes para efectos comparativos (Cleveland 1993) e incluso arbitrarios (Shennan 2006). Baxter ha indicado que las EDK son excelentes alternativas ya que proveen de una "representación menos gruesa de los datos, y a diferencia del histograma, la apariencia del EDK no depende de la elección del punto de inicio del análisis" (Baxter et al. 1997), por consiguiente se evita la arbitrariedad del histograma. En este artículo presento una alternativa al uso de histogramas, no solamente como un modo de presentación de datos, sino también como una herramienta analítica espacial con la finalidad de comprender el comportamiento de 3020 fragmentos cerámicos diagnósticos y del mismo modo identificar cómo este comportamiento está estructurado y organizado en un espacio determinado. Este método estadístico de densidad no paramétrica es relativamente ineficiente para muestras pequeñas, siendo esta la razón por la cual es necesaria una aproximación selectiva que tome en cuenta el tamaño de la muestra a fin de evitar la fabricación de modos estadísticos.

Se han utilizado análisis EDK univariados y bivariados con el propósito de identificar si los modos identificados en EDK univariados son replicados cuando otra unidad de medida es agregada. Una de las dificultades principales de este método es su marcada ausencia en la mayoría de programas estadísticos disponibles (Shennan 2006) por lo que su uso está limitado. Sin embargo, recientemente tanto software comercial como open source están incluyendo rutinas EDK dentro de las opciones disponibles por lo que es de esperar que este artículo contribuya a la utilización de este método el cual, como se verá, es una valiosa herramienta de análisis estadístico. El registro arqueológico utilizado en este artículo proviene de las excavaciones realizadas por el autor en el sitio arqueológico de Chavín de Huántar.

\section{El Sitio}

El centro ceremonial de Chavín de Huántar está localizado en la provincia de Huari, departamento de Ancash, a 3200 msnm, en el tinkuy formado por los ríos Wacheqsa y Mosna (Fig. 1). Desde los trabajos de Julio C. Tello en 1919, Chavín ha sido un referente importante en la discusión de los orígenes de la complejidad social en los Andes, ya sea como una cultura madre (Tello 1942, 1943, 1960), como un desarrollo complejo derivado del iniciado en Mesoamérica (Uhle 1902), como una síntesis de procesos sociales costeños y serranos (Burger 1993; Burger 1992, 1998) o como un lugar en el cual la autoridad era construida, materializada y transmitida (Kembel y Rick 2004; Rick 2005, 2006, 2008). Las investigaciones del autor se desarrollaron en el sector Wacheqsa (Mesia 2007), localizado inmediatamente al norte del area monumental (Fig. 2). A través de un cuidadoso programa de muestreo arqueológico y una larga pero productiva reconstrucción tridimensional estratigráfica, se han podido identificar cinco unidades espaciales prehistóricas (Fig. 3), que comprenden 700 años divididos en dos fases prehistóricas (Tabla 1). Antes de discutir los análisis EDK, considero importante discutir brevemente la naturaleza de las unidades espaciales identificadas -en las cuales se recuperó la muestra de 3020 fragmentos analizados en el presente artículo- a fin de comprender los resultados presentados en la sección final.

Tabla 1: Cronología del Sector Wacheqsa, Chavín de Huántar

\begin{tabular}{|l|l|l|}
\hline Fase & Fechas & Unidades especiales \\
\hline Janabarriu & $800-550$ ANE & Basural, Cuartos de Piedra, Plataformas Tardías \\
\hline Urabarriu & $1200-800$ ANE & Correntera de Agua, Plataformas Tempranas \\
\hline
\end{tabular}




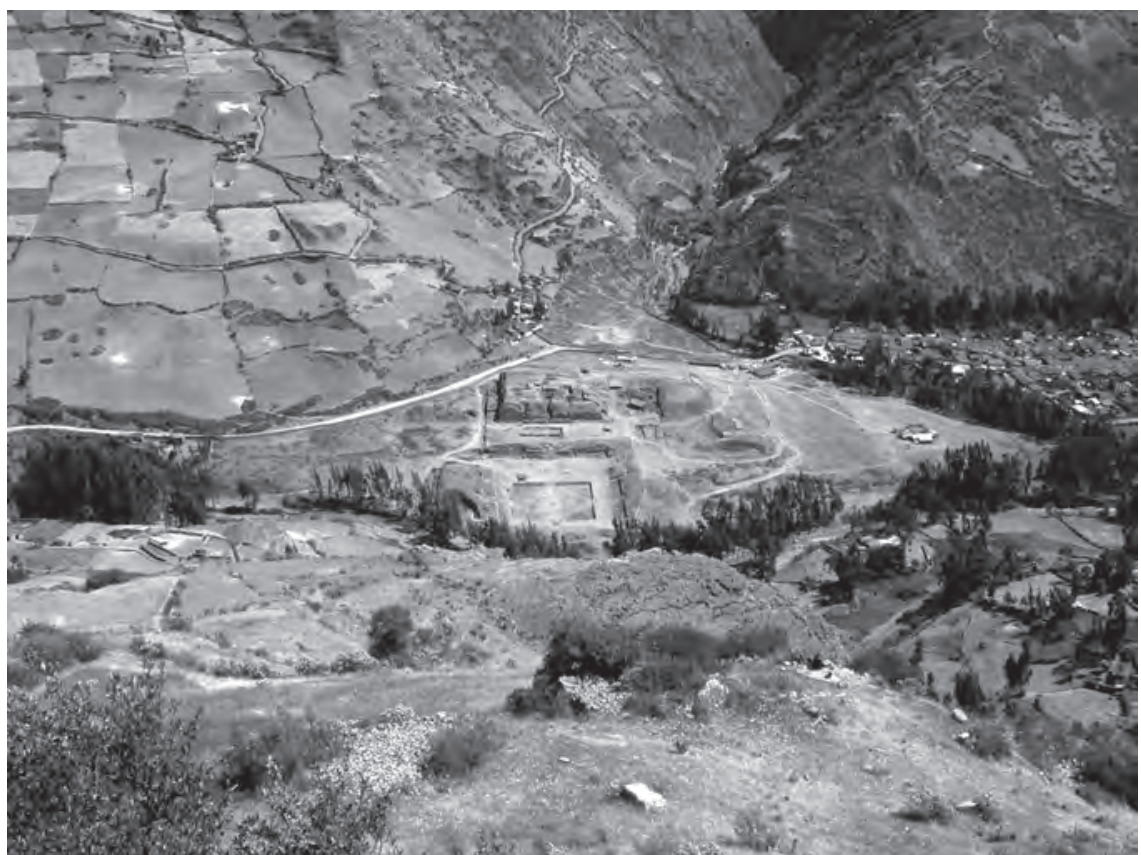

Figura 1. Chavín de Huántar visto desde lo alto de la margen derecha del río Mosna. (Foto: John Wolf).

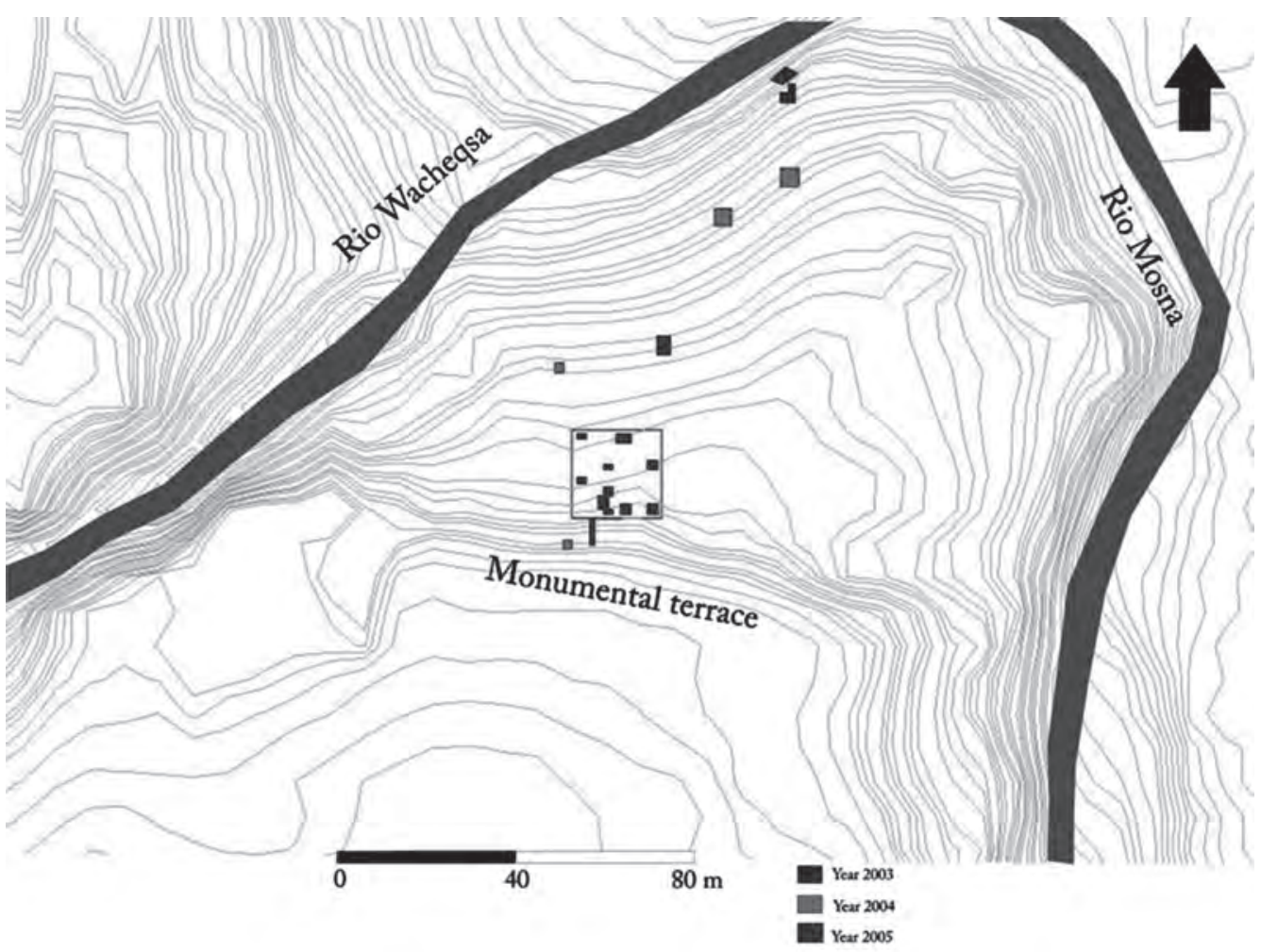

Figura 2. Plano de las excavaciones realizadas en el Sector Wacheqsa, Chavín de Huántar. 


\subsection{Unidades espaciales en el sector Wacheqsa}

La fase más antigua identificada en el sector Wacheqsa es la fase Urabarriu, la cual cubre los 1200-800 ANE (Mesia 2007; Rick et al. 2010). Está compuesta por las siguientes unidades espaciales: Correntera de agua (CA) y Plataformas Tempranas (PT). CA está localizada en el extremo sur del sector Wacheqsa y comprende un área estimada de $48 \mathrm{~m}^{2} \mathrm{y}$ un volumen estimado de $46 \mathrm{~m}^{3}{ }^{1}$. La profundidad promedio de esta unidad espacial es de 3,05 $\mathrm{m}$ bajo superficie. Se caracterizó por una sucesión de niveles compactos de arena gris y verdosa alternados con grava fina y gruesa. Se excavaron en total 5,72 $\mathrm{m}^{3}$, recuperándose 330 elementos arqueológicos con una densidad promedio de 57 elementos por $\mathrm{m}^{3}$. La cerámica identificada en esta unidad se asemejan a las identificadas como Urabarriu por Burger (1984, 1998); no se encontró fragmento cerámico alguno relacionado o similar a Janabarriu. PT es una unidad localizada en las secciones norte y central del Sector Wacheqsa. Comprende un área estimada de 1100 $\mathrm{m}^{2}$ y una profundidad promedio de 2,04 $\mathrm{m}$. Se caracteriza por una secuencia de depósitos con muy pequeña inclinación, con superficies compuestas por rocas angulares pequeñas y una baja densidad de materiales arqueológicos. Se excavó un total de 20,3 $\mathrm{m}^{3}$, recuperándose 978 elementos arqueológicos con una densidad promedio de 48 elementos por metro cúbico. Similar a CA, la cerámica recuperada en esta unidad se relaciona a la llamada Urabarriu (Fig. 4).

La siguiente fase es Janabarriu, la cual data entre los 800-500 ANE (Mesia 2007; Rick et al. 2010) (Fig. 5). Está compuesta por las siguientes unidades espaciales: Basural (B), Plataformas Tardías (PTA) y Cuartos de Piedra (CP). El Basural está localizado en el extremo sur del sector Wacheqsa, sobre la unidad CA, comprendiendo un área estimada de $48 \mathrm{~m}^{2}$, un volumen estimado de $83 \mathrm{~m}^{3}$, con casi $2 \mathrm{~m}$ de grosor promedio. Se caracteriza por capas compactas y semicompactas mezcladas con rocas angulares y cantos rodados grandes y por una alta densidad de elementos arqueológicos. Se excavó un total de 22,03 $\mathrm{m}^{3}$, recuperándose 15814 elementos arqueológicos, obteniéndose una densidad general de 718,18 elementos por $\mathrm{m}^{3}$. La unidad PTA está localizada en la sección centro-sur del sector Wacheqsa y comprende un área estimada de $223 \mathrm{~m}^{2}$ con un volumen también estimado de $276 \mathrm{~m}^{3}$; se caracteriza por presentar una matriz compacta con abundantes rocas angulares y cantos rodados medianos y grandes, además de una baja densidad de elementos arqueológicos. Se excavó un total de 5,88 $\mathrm{m}^{2}$, recuperándose 102 elementos arqueológicos, obteniéndose una densidad de 17,3 elementos por metro cuadrado. La unidad CP está localizada en la sección central del sector Wacheqsa, directamente sobre la unidad PT, y comprende un área estimada de $1717 \mathrm{~m}^{2}$ y un volumen igualmente estimado de 668,47 $\mathrm{m}^{3}$. Presenta cuartos de piedra con pasajes interconectados, los cuales fueron tapados con un relleno suelto de cantos rodados y piedras angulares. Una vez que los cuartos y pasajes estuvieron cubiertos, se revistió la zona con una capa de piedras cuadrangulares de tamaño mediano, que formaron la superficie de extensa plataforma sobre los cuartos y pasillos. Se excavó un total de $22,3 \mathrm{~m}^{2}$ del que se recuperaron 3425 elementos arqueológicos, obteniéndose una densidad total de 154 elementos por metro cuadrado.

\section{Estimados de Densidad Kernel}

Una muestra de 3020 fragmentos diagnósticos de un total de 12017 fue tabulada bajo los siguientes rubros: Estrato, Unidad Espacial, Forma, Diámetro, Grosor y Fase, con la finalidad de identificar patrones de asociación entre las categorías de diámetro y grosor por tipo cerámico y a su vez encontrar o descartar diferencias entre los patrones identificados por unidad espacial. Se identificaron los siguientes tipos cerámicos: olla sin cuello, cuenco, jarra, vaso y plato. Los análisis EDK sirvieron para identificar modos en diámetros y grosores por tipo en cada unidad espacial. Los EDK pueden ser segregados para los propósitos de este artículo en univariados y bivariados. Un EDK univariado puede ser entendido

1 Estas y otras medidas similares fueron obtenidas a partir de la reconstrucción tridimensional estratigráfica realizada en CAD-LAND [17] 


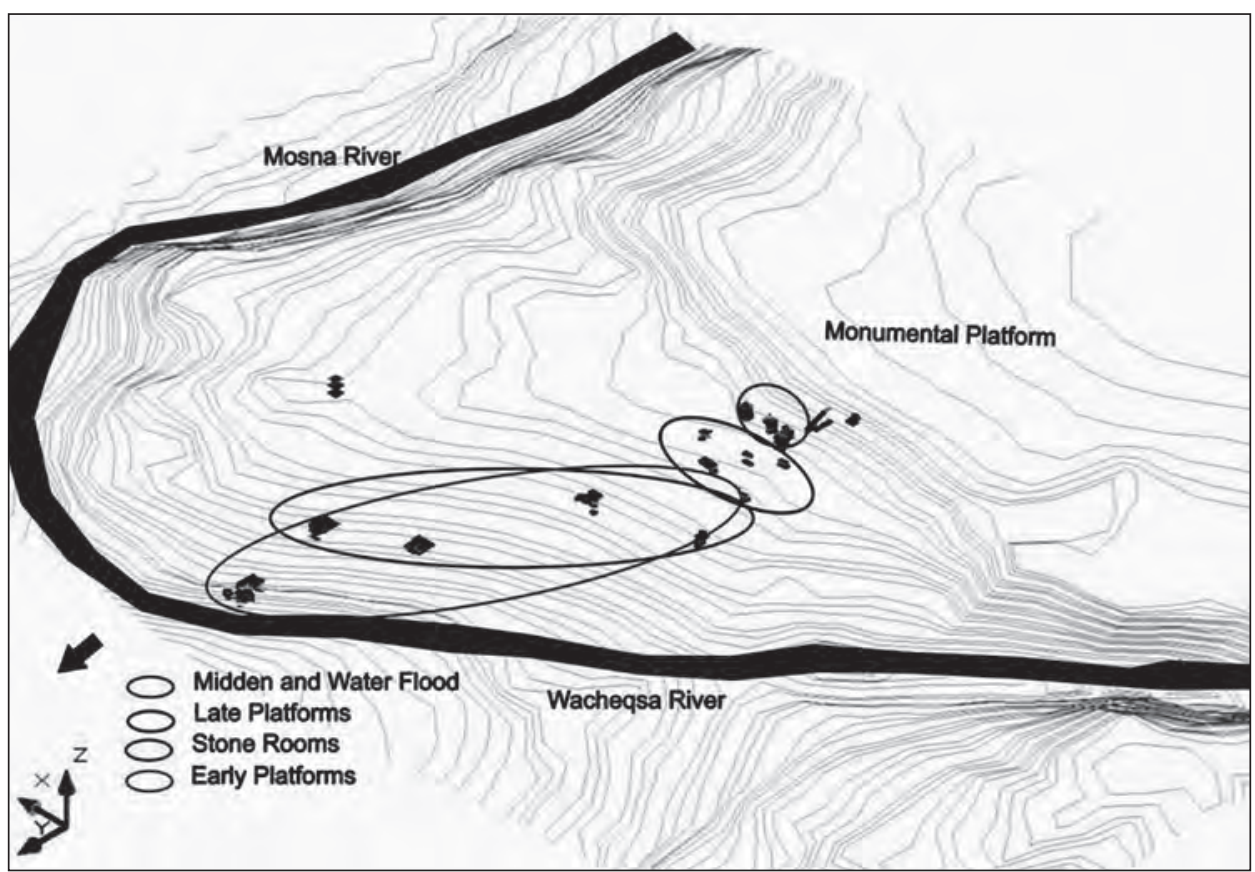

Figura 3. Topografía y unidades espaciales identificadas en el Sector Wacheqsa, Chavín de Huántar.

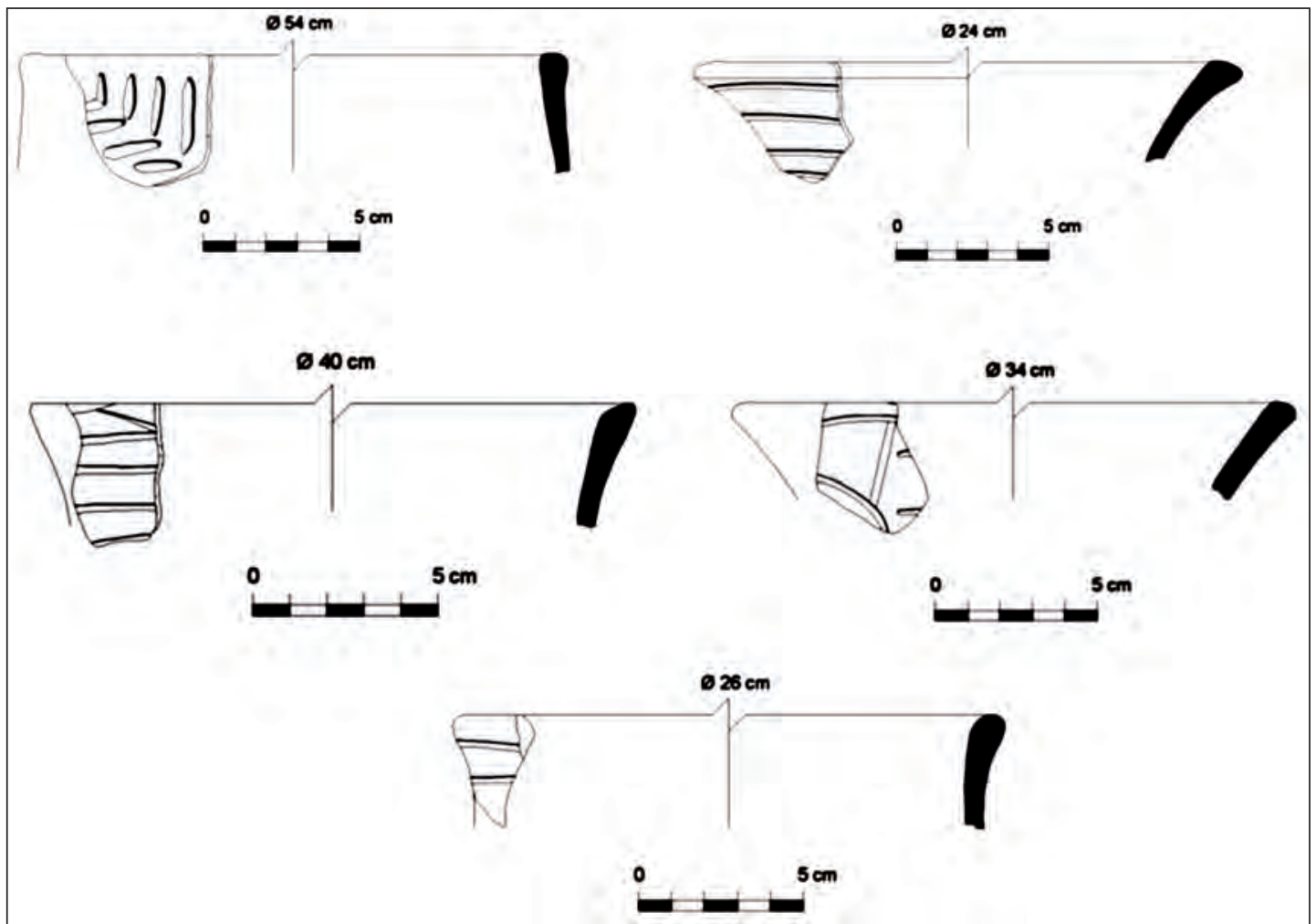

Figura 4. Cerámica de la Fase Urabarriu (1200 - 800 ANE) recuperada en el Sector Wacheqsa, Chavín de Huántar. 

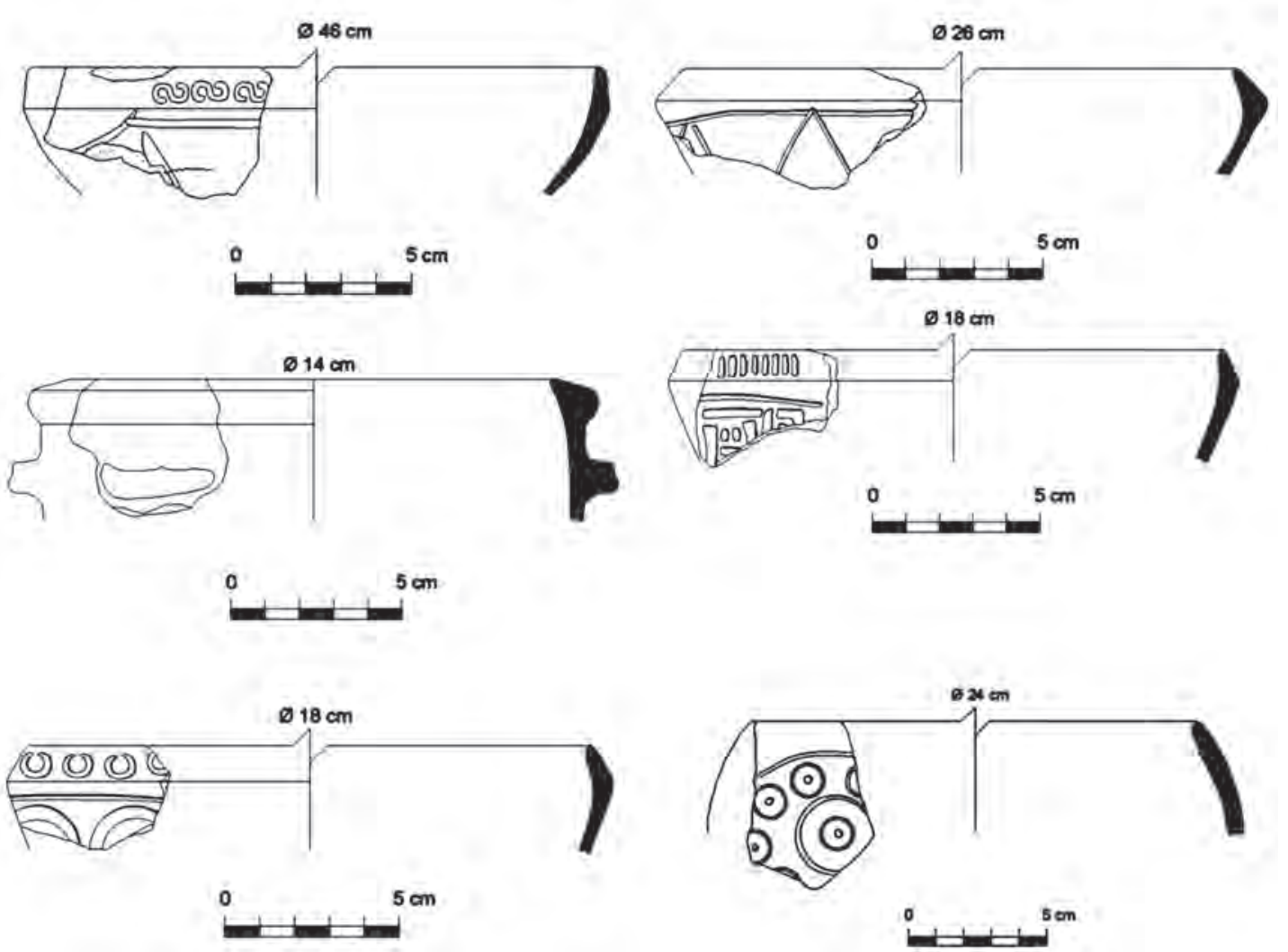

Figura 5. Cerámica de la Fase Janabarriu (800 - 550 ANE) recuperada en el Sector Wacheqsa, Chavín de Huántar.

como un histograma liso que evita las restricciones de un histograma regular (Baxter 2003; Baxter et al. 1997; Shennan 2006; Wand y Jones 1995). Dado $n$ puntos X1, X2,..., Xn situados en una recta, un EDK puede ser obtenido colocando un salto (esencialmente una función de densidad unimodal) en cada punto, sumando luego la altura de cada salto en cada punto, en el eje X. El kernel es usualmente una probabilidad simétrica de función de densidad (Baxter et al. 1997). La extensión del salto está determinada por una extensión de banda (band-width), que es análoga a la extensión de compartimento (bin-width) de un histograma. El tamaño del la extensión de banda es muy importante ya que determinará el resultado de la estimación. No existe una teoría uniforme sobre cómo regular la extensión de banda, por lo que es apropiado hacerla de modo intuitivo, intentando encontrar el balance entre resultados under smoothed y over smoothed (Baxter 2003; Baxter et al. 1997; Shennan 2006). Con la finalidad de poder lograr este balance, es necesario "iniciar con una extensión de banda grande y disminuir el tamaño de la misma hasta que fluctuaciones más azarosas que estructurales empiezan a aparecer" (Wand y Jones 1995).

Si los EDK univariados son observados como alternativas al histograma, "podría argumentarse que con el dato univariado, la ventaja de usar EDK sobre un histograma para presentaciones de datos no son tan grandes como para ser utilizadas a menudo. Para el caso análisis bivariados, el uso de EDK es más efectivo y marca la diferencia" (Baxter et al. 1997). El potencial de los análisis EDK para datos bivariados es muy alto; los EDK son muy efectivos cuando son aplicados a diagramas de dispersión, mostrando concentraciones de puntos o modos en los datos, especialmente para muestras extensas. Los EDK bivariados son eficientes delineando contornos de inclusión de acuerdo a porcentajes específicos en puntos densamente agrupados; EDK puede ser utilizado como un método informal de agrupamiento que no impone una estructura artificial 
al dato como lo hacen otros métodos formales (Wand y Jones 1995). Los EDK bivariados grafican una superficie lisa que describe qué tan densos se encuentran los puntos sobre la superficie, esos gráficos pueden utilizarse para producir gráficos de contorno que conducen a representaciones gráficas de los datos examinados (Baxter et al. 1997). En el presente artículo los gráficos EDK univariados de diámetros y grosores fueron realizados por tipo de vasija en cada unidad espacial, utilizando el software estadístico R, lo mismo que los gráficos bivariados. La secuencia de códigos de programación se incluye como apéndice al final del presente trabajo.

Con la finalidad de utilizar una medida de control adicional a los resultados establecidos por las rutinas EDK se ha utilizado el programa JMP en donde se produjeron tablas modales bivariadas que luego fueron confrontadas con los gráficos EDK. Sobre la base de rutinas EDK, JMP realiza un análisis de agrupamiento (modal cluster) en donde las mayores concentraciones o modos son trasladados a una tabla en donde se hace una identificación definitiva de modalidades. JMP divide los axis de diámetro y grosor en intervalos de 50 marcas o compartimientos de un total de 2500 , cuenta los puntos entre cada marca, decide la desviación standard kernel a utilizar siguiendo las recomendaciones de Foster (Bowman y Foster 1992) y realiza una rutina EDK utilizando el algoritmo de transformación rápida Fourier (FFT por sus siglas en ingles). Estos modos son los que representan el comportamiento cerámico de acuerdo a asociaciones de diámetro y grosor y sirven como un importante instrumento de control en la verificación de los resultados obtenidos en rutinas EDK en R.

Es importante mencionar que la decisión de trabajar con EDK univariados y bivariados recae en examinar si los modos observados en EDK univariados son replicados utilizando EDK bivariados con la adición de grosor a diámetro. Del mismo modo era importante examinar si diámetro es un buen indicador de modalidad al investigar tamaños de vasijas tal cual lo podría indicar su extenso uso en la literatura arqueológica (Blitz 1993; Drennan 1996; Longacre 1999; Mills 1999; Potter 2000; Rosenswig 2007). Es de esperar que los modos reflejados en el análisis univariado resistan la adición de la categoría de grosor.

\section{Resultados}

Una muestra de 3020 fragmentos diagnósticos fue utilizada para generar EDK univariados y bivariados de diámetros de bordes y grosores de cuellos con la finalidad de identificas patrones de distribución y variación de vasijas cerámicas al interior de las unidades espaciales descritas previamente. La tabla $2^{2}$ muestra la variación de la muestra por unidad espacial, mientras que en la tabla 3 se observa la variación de tipos cerámicos por unidad espacial. La tabla 4 presenta los datos de la tabla 3 en porcentajes. Es interesante notar que PT y PTA están caracterizados por la preponderancia de ollas sin cuello, mientras que en $\mathrm{B}$, CP y CA son los cuencos los más ubicuos. Las jarras conforman el tercer tipo prevalente en todas las unidades mientras que el cuarto está conformado por botellas. Vasos y platos tienen

Tabla 2: Variación de la muestra por unidad espacial.

\begin{tabular}{|l|r|}
\hline \multicolumn{1}{|c|}{ Unidad Espacial } & \multicolumn{1}{c|}{ n } \\
\hline Plataformas Temprana & 118 \\
\hline Plataformas Tardías & 42 \\
\hline Basural & 2441 \\
\hline Cuartos de piedra & 317 \\
\hline Correntera de agua & 102 \\
\hline
\end{tabular}
un bajo porcentaje y son casi inexistentes en la muestra.

Con esta distribución elemental de vasijas, el siguiente paso fue el de reconocer patrones específicos de variabilidad diámetro/grosor en las unidades espaciales. Con la finalidad de reconocer estos patrones se utilizaron las agrupaciones de modos bivariados (modal clusters) extraídos con el software JMP. Como se explicó anteriormente, se utilizaron rutinas EDK univariadas y bivariadas debido a la

2 Los gráficos se pueden leer del siguente modo: Midden=Basural, Early Platforms=Plataformas Tempranas, Late Platforms=Plataformas Tardías, Stone Rooms= Cuartos de Piedra, Water Flood=Correntera de Agua. OSC=Ollas sin cuello, Bowls= Cuencos, Jars=Jarras, Bottles=Botellas, Cups=Vasos y Plates=Platos. 
Tabla 3: Variación de tipos cerámicos por unidad espacial.

\begin{tabular}{|l|r|r|r|r|r|r|r|}
\hline Unidad Espacial & Cuencos & OSC & Jarras & Botellas & Vasos & Platos & Total \\
\hline Plataformas Temprana & 36 & 58 & 16 & 5 & 0 & 2 & 118 \\
\hline Plataformas Tardías & 14 & 20 & 6 & 1 & 1 & 0 & 42 \\
\hline Basural & 1263 & 656 & 338 & 101 & 37 & 46 & 2441 \\
\hline Cuartos de piedra & 143 & 111 & 51 & 4 & 1 & 7 & 317 \\
\hline Correntera de agua & 40 & 37 & 14 & 8 & 1 & 2 & 102 \\
\hline Total & 1496 & 882 & 425 & 119 & 40 & 57 & 3020 \\
\hline
\end{tabular}

Tabla 4: Variación porcentual de tipos cerámicos por unidad espacial.

\begin{tabular}{|l|r|r|r|r|r|r|}
\hline Unidad Espacial & \multicolumn{1}{|c|}{ Cuencos } & \multicolumn{1}{c|}{ OSC } & \multicolumn{1}{c|}{ Jarras } & \multicolumn{1}{c|}{ Botellas } & \multicolumn{1}{c|}{ Vasos } & \multicolumn{1}{c|}{ Platos } \\
\hline Plataformas Temprana & 30,51 & 49,15 & 13,56 & 5,08 & 0,00 & 1,69 \\
\hline Plataformas Tardías & 33,33 & 47,62 & 14,29 & 2,27 & 2,38 & 0,00 \\
\hline Basural & 51,74 & 26,87 & 11,19 & 3,34 & 1,52 & 1,88 \\
\hline Cuartos de piedra & 45,11 & 36,27 & 16,09 & 1,26 & 0,32 & 2,21 \\
\hline Correntera de agua & 39,22 & 36,26 & 13,73 & 7,84 & 0,98 & 1,96 \\
\hline
\end{tabular}

necesidad de analizar si los modos identificados en EDK univariados eran replicados utilizando el grosor en adición al diámetro. Se predijo que las modalidades reflejadas con la categoría de diámetro resistirían la inclusión del grosor como dato analítico. Los resultados indicaron que cuando se agrega grosor, nuevos modos ligeramente diferentes aparecen. El grosor actúa como una medida de control agrupando modos de acuerdo a asociaciones más robustas ente diámetro y grosor. Las siguientes secciones del presente artículo presentan un conjunto de datos organizados en tablas y gráficos. Las tablas pueden ser consideradas como estándar mientras que los gráficos están organizados de acuerdo a los siguientes tipos: EDK univariados, EDK bivariados en dos dimensiones y EDK bivariados en tres dimensiones. Los gráficos univariados se explican por si solos, mientras que los gráficos bivariados en tres dimensiones lucen diferentes a los de dos dimensiones lo cual motiva a proponer que los análisis EDK bivariados en realidad enfrentan problemas tridimensionales antes que bidimensionales. La extensión de banda, es mejor apreciada en términos tridimensionales antes que bidimensionales, pero esto es mejor observado en los gráficos que ilustran el presente artículo.

\subsection{OLLAS SIN CUELLO}

Se tiene una muestra total de 751 fragmentos de ollas sin cuello. La tabla 5 muestra los resultados univariados por diámetro y grosor. Las figuras 6 y 7 presentan estos modos y cómo se sobreponen. Cuando estas dos categorías son trasladadas a un gráfico EDK bivariado, tres modos aparecen, como se observa en la tabla 5 y en las figuras 8 y 9. La tabla 6 muestra la población total de tipos de ollas sin cuello. La prevalencia de ollas sin cuello en el sector Wacheqsa se da en aquellas de tamaño grande, sin embargo, existe otro modo, el de las ollas medianas. Conservadoramente dos grandes tamaños de ollas sin cuello en la muestra examinada. A fin de examinar variaciones específicas en las unidades espaciales, las ollas sin cuello serán segregadas de acuerdo a ellas.

\subsubsection{Basural}

Existen 549 fragmentos de ollas sin cuello en la muestra examinada. La tabla 5 ilustra los resultados de EDK univariados tanto en diámetro como en grosor. Cuando estos datos son trasladados a un gráfico EDK bivariado, tres modos aparecen tal cual se observa en la tabla 5 y en las figuras 8 y 9 , lo cual 
Christian Mesía / Uso de estimados de densidad Kernel en la investigación de grupos cerámicos...

Tabla 5: Agrupaciones modales univariadas y bivariadas de Ollas Sin Cuello.

\begin{tabular}{|c|c|c|c|}
\hline \multicolumn{4}{|c|}{ AGRUPACIONES MODALES UNIVARIADAS } \\
\hline KDE & Mode 1 & Mode 2 & Mode 3 \\
\hline Diámetro & $15 \mathrm{~cm}$ & $25 \mathrm{~cm}$ & Ausente \\
\hline Grosor & $0,5 \mathrm{~cm}$ & $1,2 \mathrm{~cm}$ & Ausente \\
\hline \multicolumn{4}{|l|}{ Basural } \\
\hline $\mathrm{KDE}$ & Mode 1 & Mode 2 & Mode 3 \\
\hline Diámetro & $10 \mathrm{~cm}$ & $30 \mathrm{~cm}$ & Ausente \\
\hline Grosor & $0,5 \mathrm{~cm}$ & $1,3 \mathrm{~cm}$ & Ausente \\
\hline \multicolumn{4}{|c|}{ Cuartos de Piedra } \\
\hline $\mathrm{KDE}$ & Mode 1 & Mode 2 & Mode 3 \\
\hline Diámetro & $15 \mathrm{~cm}$ & $25 \mathrm{~cm}$ & 40 \\
\hline Grosor & $0,6 \mathrm{~cm}$ & $1 \mathrm{~cm}$ & Ausente \\
\hline \multicolumn{4}{|c|}{ Plataformas Tempranas } \\
\hline $\mathrm{KDE}$ & Mode 1 & Mode 2 & Mode 3 \\
\hline Diámetro & $15 \mathrm{~cm}$ & $25 \mathrm{~cm}$ & Ausente \\
\hline Grosor & $0,8 \mathrm{~cm}$ & $1,2 \mathrm{~cm}$ & 1,6 \\
\hline \multicolumn{4}{|c|}{ Correnteras de Agua } \\
\hline $\mathrm{KDE}$ & Mode 1 & Mode 2 & Mode 3 \\
\hline Diámetro & $12 \mathrm{~cm}$ & $27 \mathrm{~cm}$ & Ausente \\
\hline Grosor & $0,8 \mathrm{~cm}$ & $1,2 \mathrm{~cm}$ & Ausente \\
\hline \multicolumn{4}{|c|}{ Plataformas Tardías } \\
\hline $\mathrm{KDE}$ & Mode 1 & Mode 2 & Mode 3 \\
\hline Diámetro & $13 \mathrm{~cm}$ & $20 \mathrm{~cm}$ & Ausente \\
\hline Grosor & $0,3 \mathrm{~cm}$ & $1,1 \mathrm{~cm}$ & Ausente \\
\hline
\end{tabular}

\begin{tabular}{|c|c|c|}
\hline \multicolumn{3}{|c|}{ AGRUPACIONES MODALES BIVARIADAS } \\
\hline Grosor & Diámetro & $\mathrm{n}$ \\
\hline 0,61074 & 11,68 & 60 \\
\hline 0,8906 & 14,56 & 229 \\
\hline 1,17046 & 26,08 & 436 \\
\hline \multicolumn{3}{|l|}{ Basural } \\
\hline Grosor & Diámetro & $\mathrm{n}$ \\
\hline 0,5612 & 11,68 & 97 \\
\hline 0,8888 & 15,52 & 61 \\
\hline 1,3256 & 28 & 377 \\
\hline \multicolumn{3}{|c|}{ Cuartos de Piedra } \\
\hline Grosor & Diámetro & $\mathrm{n}$ \\
\hline 0,58348 & 10,56 & 17 \\
\hline 0,95284 & 16,32 & 25 \\
\hline 1,13752 & 25,28 & 30 \\
\hline \multicolumn{3}{|c|}{ Plataformas Tempranas } \\
\hline Grosor & Diámetro & $\mathrm{n}$ \\
\hline 0,8746 & 14,6 & 37 \\
\hline 1,0412 & 24,72 & 7 \\
\hline \multicolumn{3}{|c|}{ Correntera de Agua } \\
\hline Grosor & Diámetro & $\mathrm{n}$ \\
\hline 0,7998 & 12,68 & 15 \\
\hline 1,1268 & 26,72 & 6 \\
\hline \multicolumn{3}{|c|}{ Plataformas Tardías } \\
\hline Grosor & Diámetro & $\mathrm{n}$ \\
\hline 0,735 & 14,2 & 3 \\
\hline 0,811 & 19,8 & 6 \\
\hline 1,02 & 11,12 & 5 \\
\hline
\end{tabular}
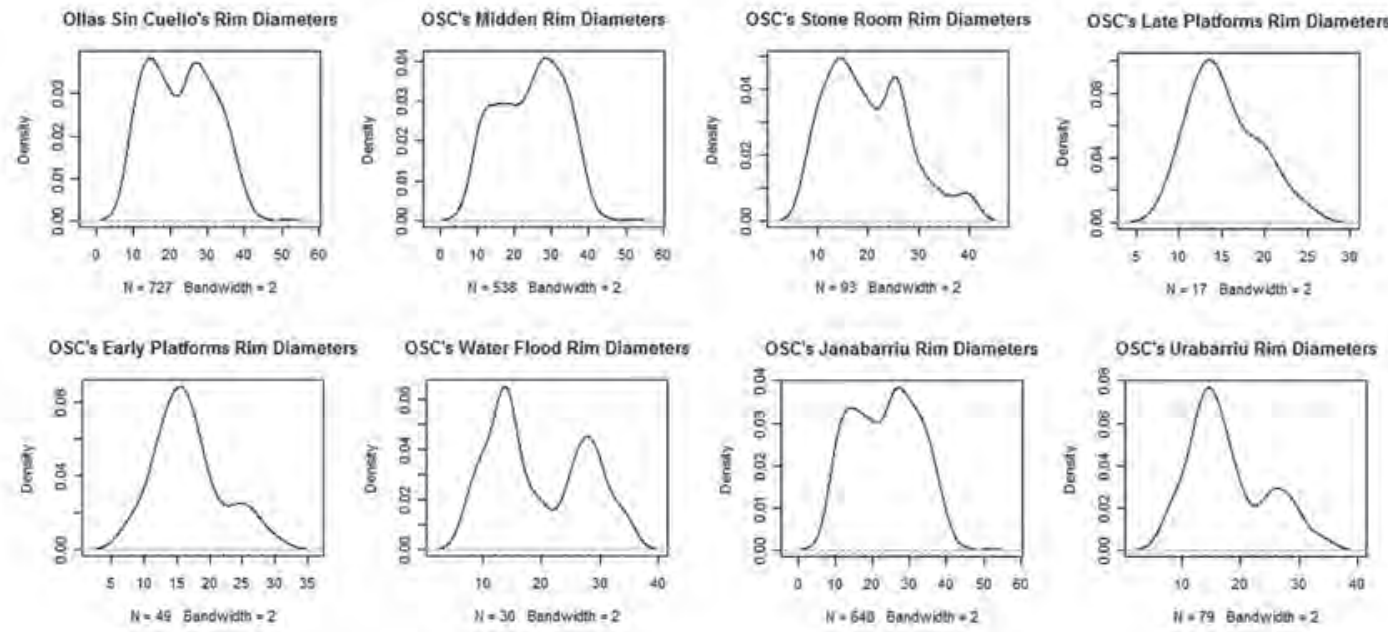

Figura 6. EDK univariados de diámetros de Ollas Sin Cuello. 

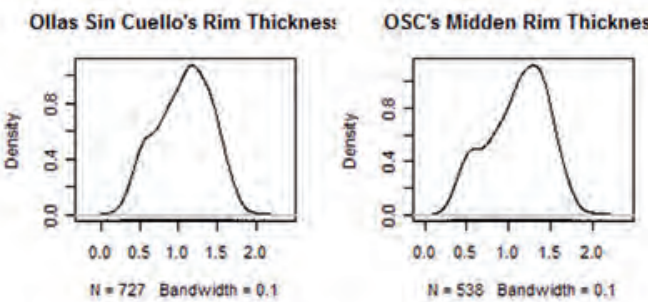

OSC's Stone Room Rim Thicknes

OSC's Late Platforms Rim Thickne

OSC's Early Platforms Rim Thicknt

OSC's Water Flood Rim Thicknes
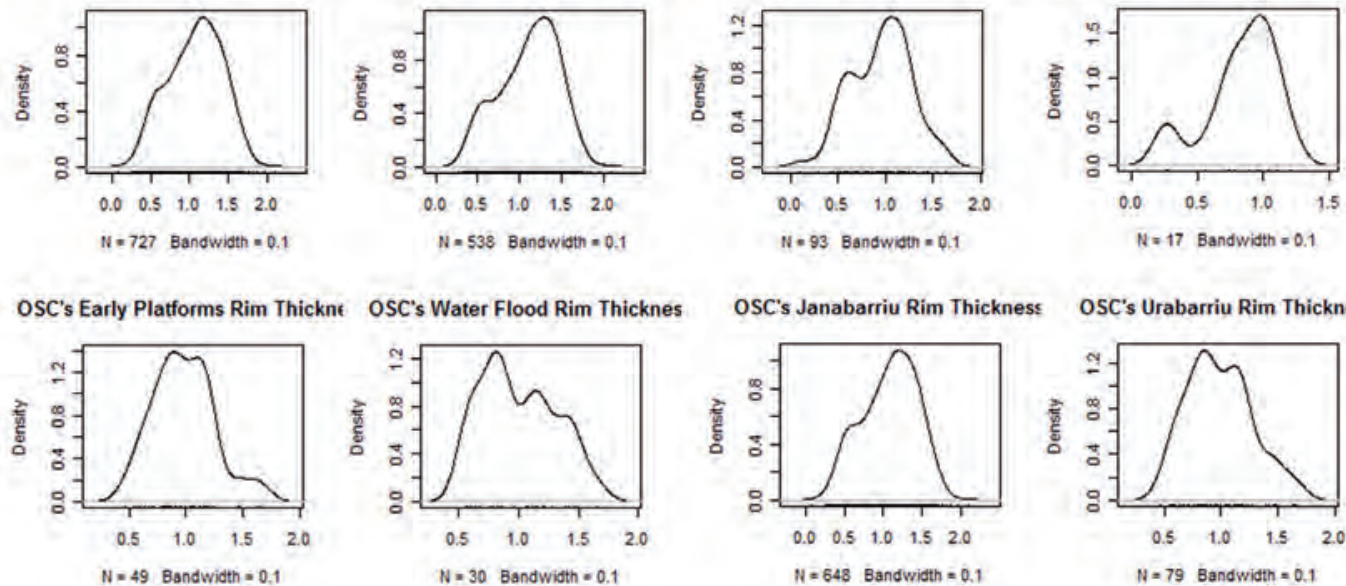

OSC's Janabarriu Rim Thickness

OSC's Urabarriu Rim Thickness
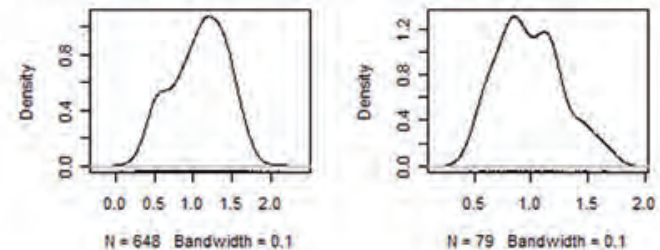

Figura 7. EDK univariados de grosores de Ollas Sin Cuello.

se ve más claramente en el gráfico tridimensional de la figura 9. La meseta observada en el gráfico bidimensional, es identificada aquí como un pico independiente. Tres tipos de ollas sin cuello han sido identificados en esta unidad analítica: pequeñas, medianas y grandes, cada una de ellas asociadas con medidas de grosor particulares que se incrementan de acuerdo a la extensión del diámetro (tabla 6). La preponderancia de tamaño se da en ollas grandes.

\subsubsection{Cuartos de piedra}

Existen 103 fragmentos de olla sin cuello en la muestra analizada. La tabla 5 muestra los resultados de EDK univariados para diámetro y grosor. Las figuras 6 y 7 ilustran la organización de los modos univariados identificados. Los análisis KDE bivariados indican la presencia de tres modos tal cual se puede observar en la tabla 5 y en las figuras 8 y 9 . Sin embargo, como en caso de las ollas sin cuello provenientes del basural, estos modos se observan con mayor detalle en los gráficos tridimensionales. En esta unidad analítica se repiten los modos del basural (tabla 6). Hay una mayor presencia de ollas
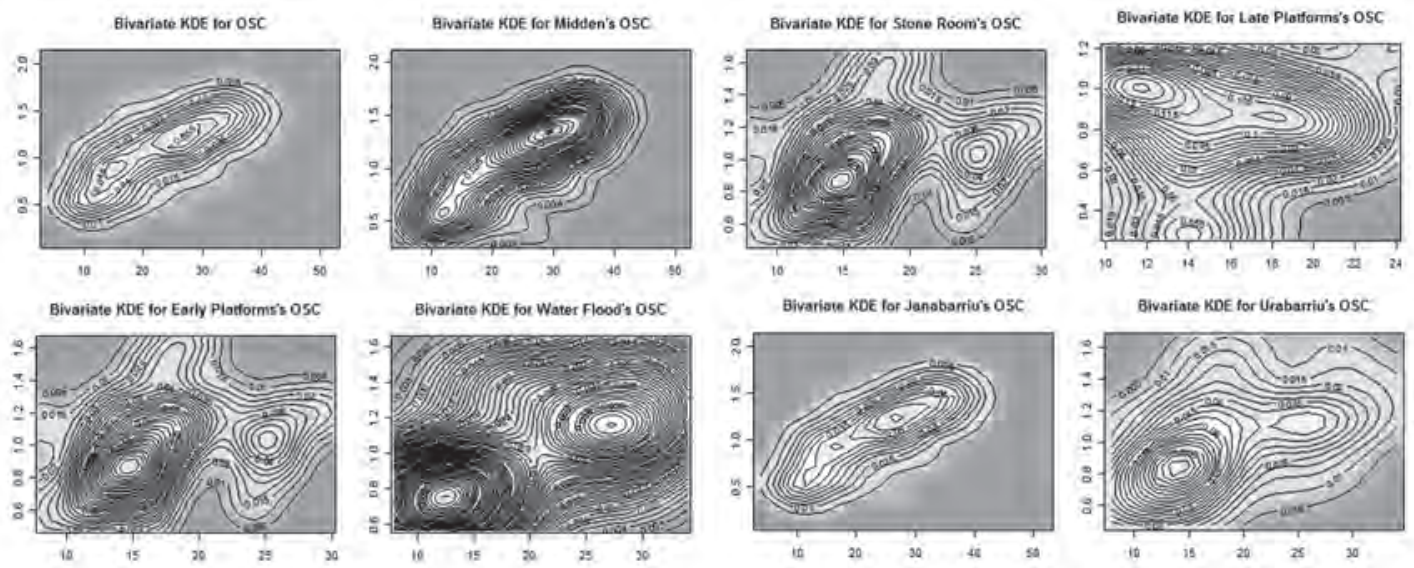

Figura 8. EDK bivariados bidimensionales de diámetros de Ollas Sin Cuello. 


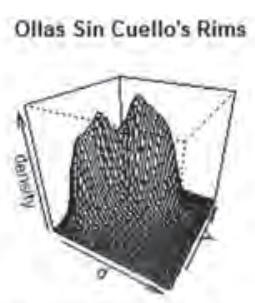

Early Platform's Ollas sin Cuellos

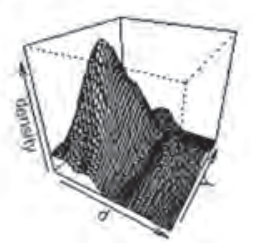

Midden's Ollas Sin Cuello

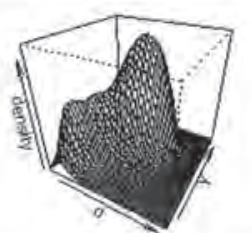

Water Flood's Ollas sin Cuellos

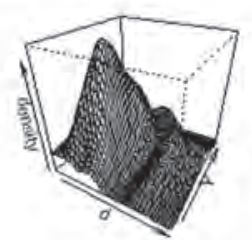

Stone Room's Ollas sin Cuellos

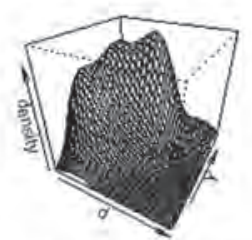

Janabarriu's Ollas sin Cuellos

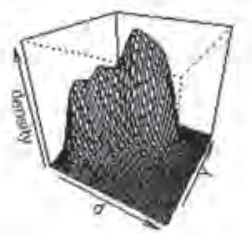

Late Platform's Ollas sin Cuellos

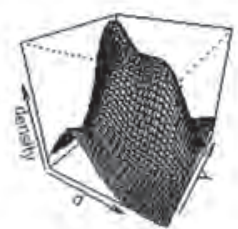

Urabarriu's Olfas Sin Cuellos

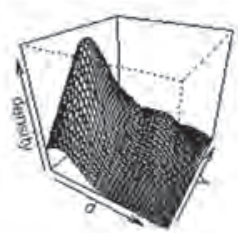

Figura 9. EDK bivariados tridimensionales de diámetros de Cuencos.

grandes y gruesas, seguidas de cerca por ollas medianas. El tercer modo observado en el examen univariado de diámetros no resistió en análisis bivariado.

\subsubsection{Plataformas Tempranas}

Existen 52 fragmentos de ollas sin cuello presentes en la muestra analizada en esta unidad analítica. La tabla 5 muestra los resultados de EDK univariados para diámetro y grosor. Las figuras 6 y 7 ilustran la organización de los modos univariados identificados. Los análisis bivariados indican la presencia de dos modos tal cual se observa en la tabla 5 y en las figuras 8 y 9. La población de ollas sin cuello en las plataformas tempranas puede ser segregada en grandes y pequeñas, con una predominancia de las últimas (tabla 6).

\subsubsection{Correntera de Agua}

Existen 30 fragmentos de ollas sin cuello presentes en la muestra analizada en esta unidad analítica. La tabla 5 muestra los resultados de EDK univariados para diámetro y grosor. Las figuras 6 y 7 ilustran la organización de los modos univariados identificados. Los análisis bivariados indican la presencia de dos modos tal cual se observa en las tabla 5 y en las figuras 8 y 9. Dos tipos de ollas sin cuello son cuantitativamente detectadas, grandes y pequeñas con preponderancia de las pequeñas. Sin embargo, es necesario tomar con cautela estos resultados ya que como lo han indicado Baxter et al. (2000), una muestra de $n=30$ es lo mínimo aceptable para EDK y aún así, los resultados deben de ser tomados con cautela. A esto se hacía referencia al comentar en la sección anterior sobre la necesidad de evitar artefactos fabricados estadísticos originados por muestras pequeñas.

\subsubsection{Plataformas Tardías}

Esta unidad especial se caracteriza por una baja densidad de materiales arqueológicos por metro cúbico. Existen 17 fragmentos de ollas sin cuello en la muestra analizada. La tabla 5 muestra los resultados de EDK univariados para diámetro y grosor. Las figuras 6 y 7 ilustran la organización de los modos univariados identificados. Los análisis bivariados indican la presencia de tres modos tal cual se observa en la tabla 5 y en las figuras 8 y 9 . Sin embargo debe de anotarse que dado lo pequeño de la muestra, los resultados son altamente preliminares. Tres modos fueron identificados, pequeños, medianos y grandes, con preponderancia de ollas sin cuello medianas (tabla 6). 
Tabla 6: Sumario de tipos y medidas de Ollas Sin Cuello.

\begin{tabular}{|l|l|l|l|}
\hline Unidad Espacial & Pequeño & Mediano & Grande \\
\hline Basural & $12 / 0,57$ & $16 / 0,9$ & $28 / 1,3$ \\
\hline Cuartos de Piedra & $11 / 0,58$ & $16 / 1,0$ & $26 / 1,1$ \\
\hline Plataformas Tempranas & $14,5 / 0,87$ & $18 / 1,5$ & $25 / 1,0$ \\
\hline Correntera de Agua & $13 / 0,80$ & Ausente & $27 / 1,1$ \\
\hline Plataformas Tardías & $11 / 1,0$ & $20 / 0,8$ & $25 / 1,1$ \\
\hline
\end{tabular}

\begin{tabular}{|l|c|c|}
\hline \multicolumn{1}{|c|}{ Unidad Espacial } & Tipos & Prevalencia \\
\hline Basural & Grande, mediano, pequeño & Grande \\
\hline Cuartos de Piedra & Grande, mediano, pequeño & Grande y mediano \\
\hline Plataformas Tempranas & Grande, y pequeño & Pequeño \\
\hline Correntera de Agua & Grande y pequeño & Pequeño \\
\hline Plataformas Tardías & Mediano y pequeño & Pequeño \\
\hline
\end{tabular}

\subsection{CUENCOS}

Se tiene una muestra total de 1334 fragmentos de ollas sin cuello. La tabla 7 muestra los resultados univariados por diámetro y grosor. Las figuras 10 y 11 presentan estos modos y como se sobreponen. Cuando estas dos categorías son trasladadas a un gráfico EDK bivariado, un modo preponderante aparece -como se observa en la tabla 7 y en las figuras 12 y 13. El modo identificado corresponde a cuencos medianos, caracterizados por un diámetro de $15,2 \mathrm{~cm}$ y un grosor de 0,43 cm (tabla 8).

\subsubsection{Basural}

La muestra consistió en 1114 fragmentos. La tabla 7 muestra los resultados de EDK univariados para diámetros y grosores mientras las figuras 10 y 11 ilustran las variaciones de modos. Cuando estas dos categorías son trasladadas a un gráfico EDK bivariado, un modo aparecen como se observa en la tabla 7 y en las figuras 12 y 13, teniéndose similar concentración a la identificada para la muestra total de cuencos. Dado que los cuencos del basural conforman el $84,66 \%$ de la muestra total, este resultado no es sorprendente.

\subsubsection{Cuartos de Piedra}

La muestra consistió en 143 fragmentos. La tabla 7 muestra los resultados de EDK univariados para diámetros y grosores mientras las figuras 10 y 11 ilustran las variaciones de modos. Cuando estas dos categorías son trasladadas a un gráfico EDK bivariado, un modo aparecen como se observa en la tabla 7 y en las figuras 12 y 13. Existe una fuerte prevalencia de cuencos medianos de 15,2 cm de diámetro y $0,50 \mathrm{~cm}$ de grosor, muy similar a lo identificado para el basural (tabla 8).

\subsubsection{Plataformas Tempranas}

La muestra consistió en 36 fragmentos. La tabla 7 muestra los resultados de EDK univariados para diámetros y grosores mientras las figuras 10 y 11 ilustran las variaciones de modos. Cuando estas dos categorías son trasladadas a un gráfico EDK bivariado, dos modos emergen como se observa en la tabla 7 y en las figuras 12 y 13. Como se observa en muestras pequeñas (aún por encima de la marca de $\mathrm{n}=30$ definida por Baxter et al.) los modos pueden ser fabricados artificialmente tanto en EDK bivariados bidimensionales y tridimensionales, más pronunciados aún en los tridimensionales. El resultado de esta unidad analítica debe de ser tomado con cautela. Los cuencos pequeños son predominantes en esta unidad espacial (tabla 8). 
Tabla 7: Agrupaciones modales univariadas y bivariadas de Cuencos.

\begin{tabular}{|l|l|l|l|}
\hline AGRUPACIONES MODALES UNIVARIADAS \\
\hline KDE & Mode 1 & Mode 2 & Mode 3 \\
\hline Diámetro & $17 \mathrm{~cm}$ & Ausente & Ausente \\
\hline Grosor & $0,5 \mathrm{~cm}$ & Ausente & Ausente \\
\hline Basural & Mode 1 & Mode 2 & Mode 3 \\
\hline KDE & $17 \mathrm{~cm}$ & Ausente & Ausente \\
\hline Diámetro & $0,4 \mathrm{~cm}$ & $0,6 \mathrm{~cm}$ & Ausente \\
\hline Grosor & Mode 1 & Mode 2 & Mode 3 \\
\hline Cuartos de Piedra & $15 \mathrm{~cm}$ & Ausente & Ausente \\
\hline KDE & $0,5 \mathrm{~cm}$ & Ausente & Ausente \\
\hline Diámetro & Mode 1 & Mode 2 & Mode 3 \\
\hline Grosor & $15 \mathrm{~cm}$ & Ausente & Ausente \\
\hline Plataformas Tempranas & Ausente \\
\hline KDE & $0,5 \mathrm{~cm}$ & $0,8 \mathrm{~cm}$ & A \\
\hline Diámetro & Mode 1 & Mode 2 & Mode 3 \\
\hline Grosor & $15 \mathrm{~cm}$ & 25 & Ausente \\
\hline Correnteras de Agua & $0,5 \mathrm{~cm}$ & $0,8 \mathrm{~cm}$ & Ausente \\
\hline KDE & \multicolumn{5}{|l|}{} \\
\hline Diámetro & Mode 1 & Mode 2 & Mode 3 \\
\hline Grosor & $15 \mathrm{~cm}$ & 25 & Ausente \\
\hline Plataformas Tardías & $0,5 \mathrm{~cm}$ & $0,8 \mathrm{~cm}$ & Ausente \\
\hline KDE &
\end{tabular}

\begin{tabular}{|l|l|l|}
\hline \multicolumn{3}{|l|}{ AGRUPACIONES MODALES BIVARIADAS } \\
\hline Grosor & Diámetro & $\mathbf{n}$ \\
\hline 0.4318 & 15,2 & 1275 \\
\hline Basural & \multicolumn{3}{|l|}{} \\
\hline Grosor & Diámetro & $\mathbf{n}$ \\
\hline 0.4318 & 15,2 & 1063 \\
\hline Cuartos de Piedra \\
\hline Grosor & Diámetro & $\mathbf{n}$ \\
\hline 0.4972 & 14,8 & 126 \\
\hline Plataformas Tempranas \\
\hline Grosor & Diámetro & $\mathbf{n}$ \\
\hline 0.53 & 10,8 & 20 \\
\hline 0.57 & 14,64 & 9 \\
\hline Correntera de Agua \\
\hline Grosor & Diámetro & $\mathbf{n}$ \\
\hline 0.3898 & 15,12 & 10 \\
\hline 0.4724 & 23,28 & 18 \\
\hline Plataformas Tardías \\
\hline Grosor & Diámetro & $\mathbf{n}$ \\
\hline 0.4928 & 17,2 & 5 \\
\hline 0.5792 & 12,16 & 4 \\
\hline
\end{tabular}
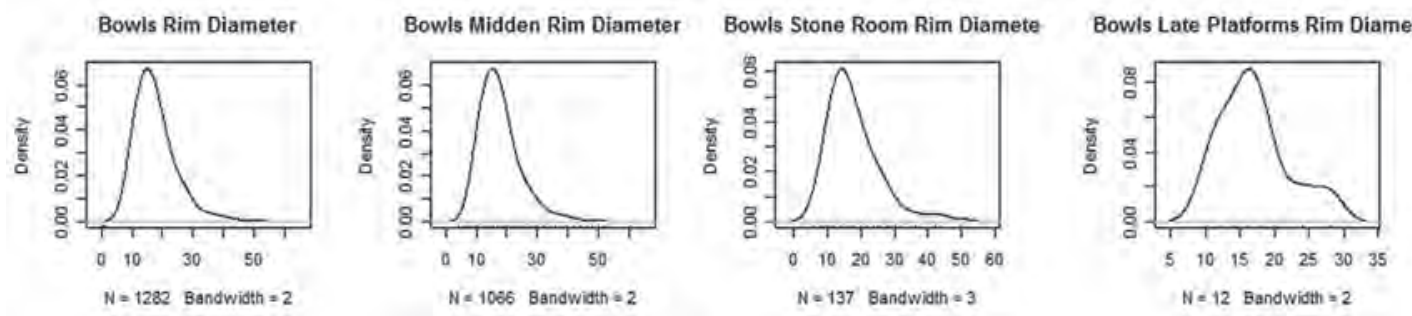

Bowls Early Platforms Rim Diamet Bowls Water Flood Rim Diamete

Bowls Janabarriu Rim Diameter

Bowls Urabarriu Rim Diameter
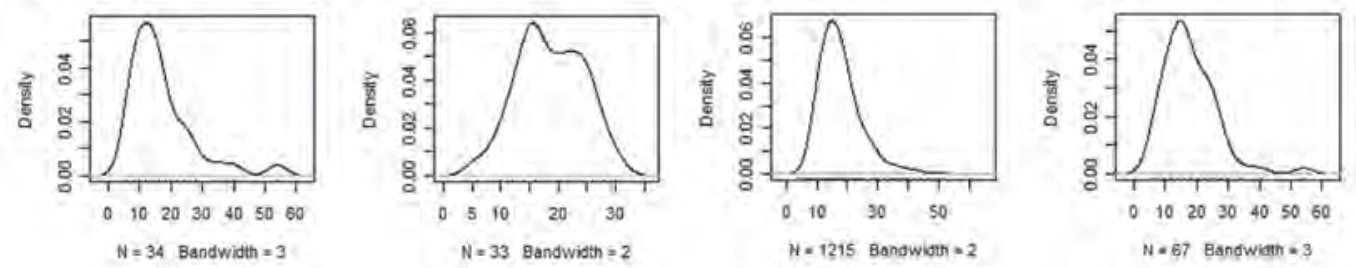

Figura 10. EDK univariados de diámetros de Cuencos.

\subsubsection{Correntera de Agua}

La muestra está conformada por 40 fragmentos. La tabla 7 muestra los resultados de EDK univariados para diámetros y grosores mientras las figuras 10 y 11 ilustran las variaciones de modos. Cuando estas dos categorías son trasladadas a un gráfico EDK bivariado, dos modos emergen como se observa en la tabla 7 y en las figuras 12 y 13. 

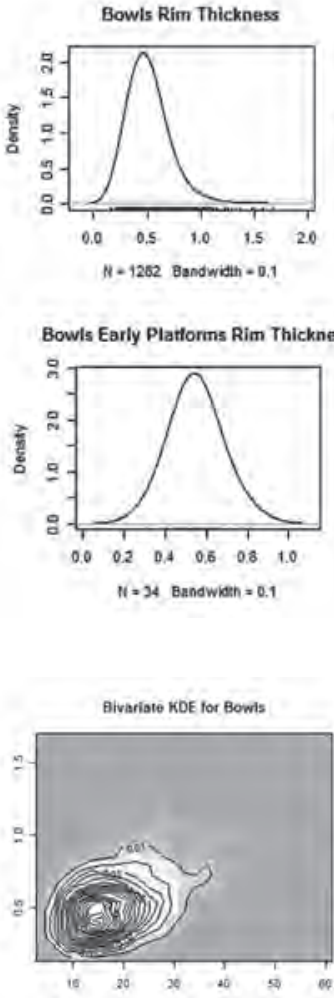

Bivariate KOE for Earty Plattorms Bowls

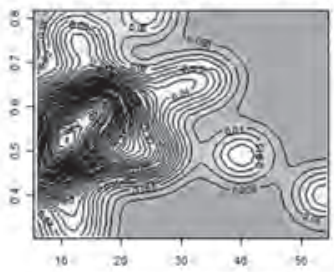

Bowls Midden Rim Thickness

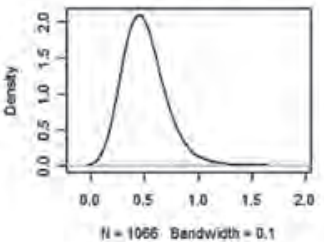

Bowls Water Flood RIm Thickness

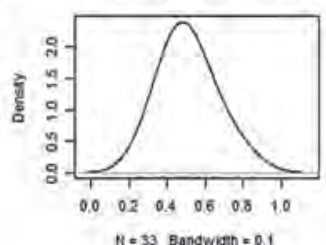

$N=\$ 3$ Bandwiath $=0.1$
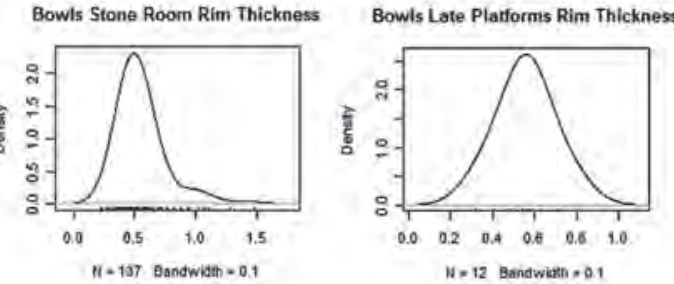

Bowis Janabartiu Rim Thickness

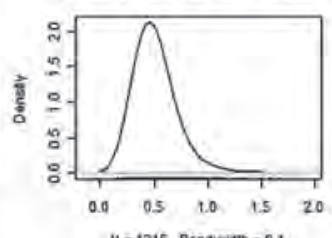

$N=1215$ Bandwatr $=0.1$

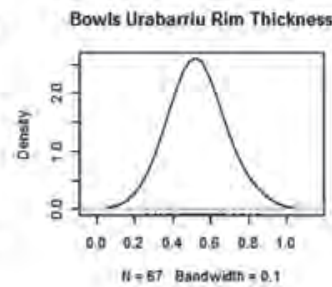

Figura 11. EDK univariados de grosores de Cuencos
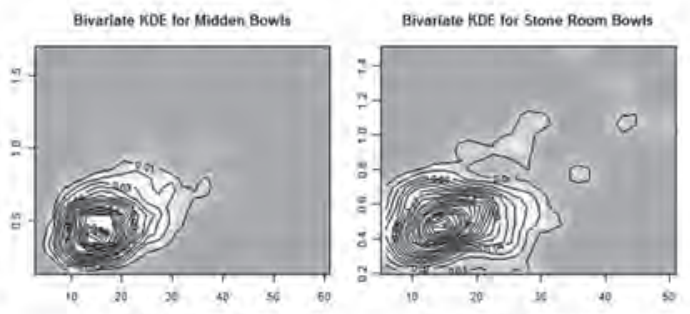

Bivariate KOE for Woter Flood Bowits
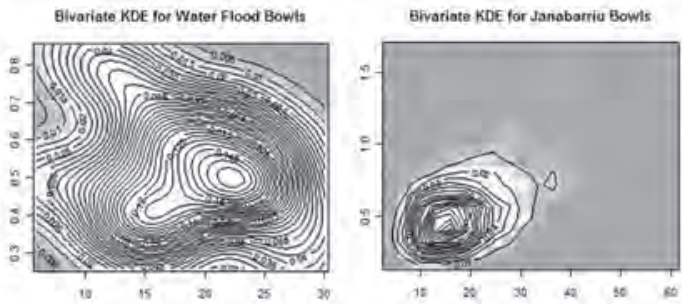

Figura 12. EDK bivariados bidimensionales de diámetros de Cuencos.

\subsubsection{Plataformas Tardías}

Muestra de 14 fragmentos. La tabla 7 muestra los resultados de EDK univariados para diámetros y grosores mientras las figuras 10 y 11 ilustran las variaciones de modos. Cuando estas dos categorías son trasladadas a un gráfico EDK bivariado, dos modos emergen como se observa en la tabla 7 y en las figuras 12 y 13. Todos los estimados de esta unidad analítica deben de ser tomados con mucha cautela debido lo pequeño de la muestra.

En la tabla 8 se puede ver un resumen de los resultados de EDK en cuencos por unidad espacial.

\subsection{JARRAS}

La muestra total de fragmentos de jarras está compuesta por 452 fragmentos. La tabla 9 muestra los resultados univariados por diámetro y grosor. Las figuras 14 y 15 presentan estos modos y como se superponen. Cuanto estas dos categorías son trasladadas a un gráfico EDK bivariado, un modo preponderante aparece, como se observa en la tabla 9 y en las figuras 16 y 17. 


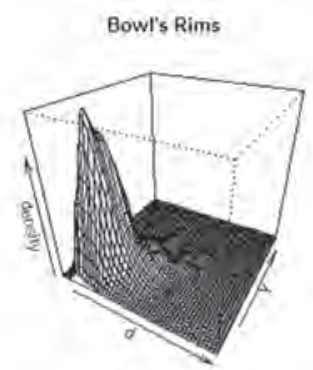

Early Platforms Bowls

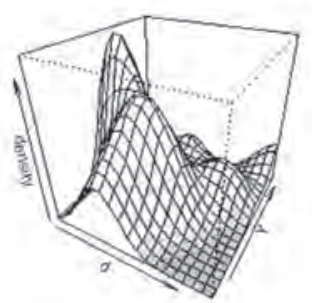

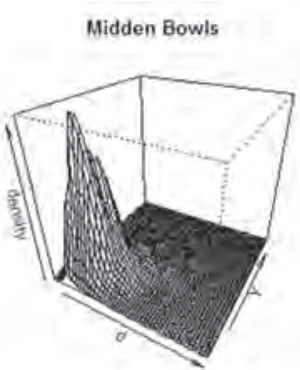

Water Flood Bowls

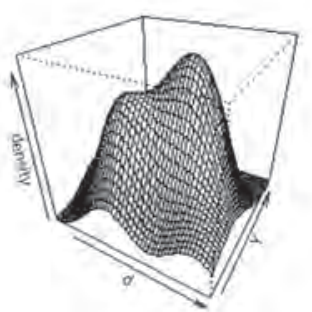

Stone Room Bowls

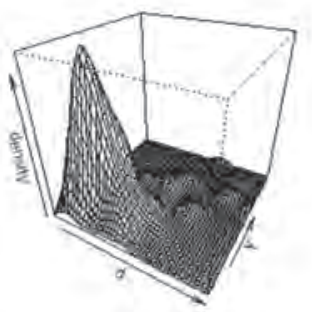

Janabarriu Bowls

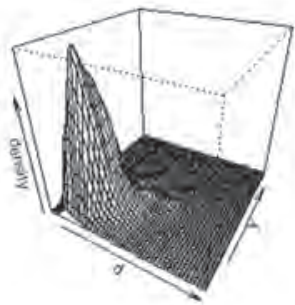

Late Platforms Bowis

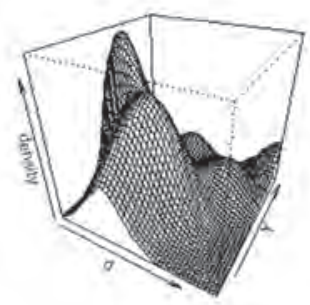

Urabarriu Bowls

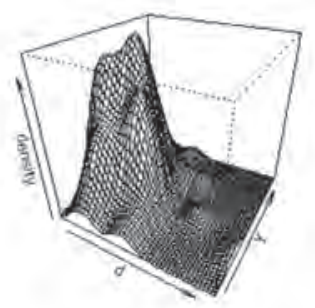

Figura 13. EDK bivariados tridimensionales de diámetros de Cuencos

\subsubsection{Basural}

La muestra total asciende a 338 fragmentos. La tabla 9 muestra los resultados univariados por diámetro y grosor. Las figuras 14 y 15 presentan estos modos y como se superponen. Cuando estas dos categorías son trasladadas a un gráfico EDK bivariado, un modo preponderante aparece -como se observa en la tabla 9 y en las figuras 16 y 17. Similar situación a la relación entre los cuencos de basural y la muestra total se repite aquí. Las jarras del basural componen el 73,7\% de la muestra total, razón por la cual los resultados son idénticos.

\subsubsection{Cuartos de Piedra}

La muestra total asciende a 51 fragmentos. La tabla 9 muestra los resultados univariados por diámetro y grosor. Las figuras 14 y 15 presentan estos modos y como se superponen. Cuando estas dos catego-

Tabla 8: Sumario de tipos y medidas de Cuencos

\begin{tabular}{|l|l|l|l|}
\hline Unidad Espacial & Pequeño & Mediano & Grande \\
\hline Basural & Ausente & $15 / 0,43$ & Ausente \\
\hline Cuartos de Piedra & Ausente & $15 / 0,50$ & Ausente \\
\hline Plataformas Tempranas & $11 / 0,52$ & $15 / 0,60$ & Ausente \\
\hline Correntera de Agua & Ausente & $15 / 0,40$ & $23 / 0.47$ \\
\hline Plataformas Tardías & $12 / 0,50$ & $17 / 0,49$ & Ausente \\
\hline
\end{tabular}

\begin{tabular}{|l|l|l|}
\hline \multicolumn{1}{|c|}{ Unidad Espacial } & \multicolumn{1}{c|}{ Tipos } & Prevalencia \\
\hline Basural & Mediano & Mediano \\
\hline Cuartos de Piedra & Mediano & Mediano \\
\hline Plataformas Tempranas & Pequeño y mediano & Pequeño \\
\hline Correntera de Agua & Mediano y grande & Grande \\
\hline Plataformas Tardías & Pequeño y mediano & Mediano \\
\hline
\end{tabular}



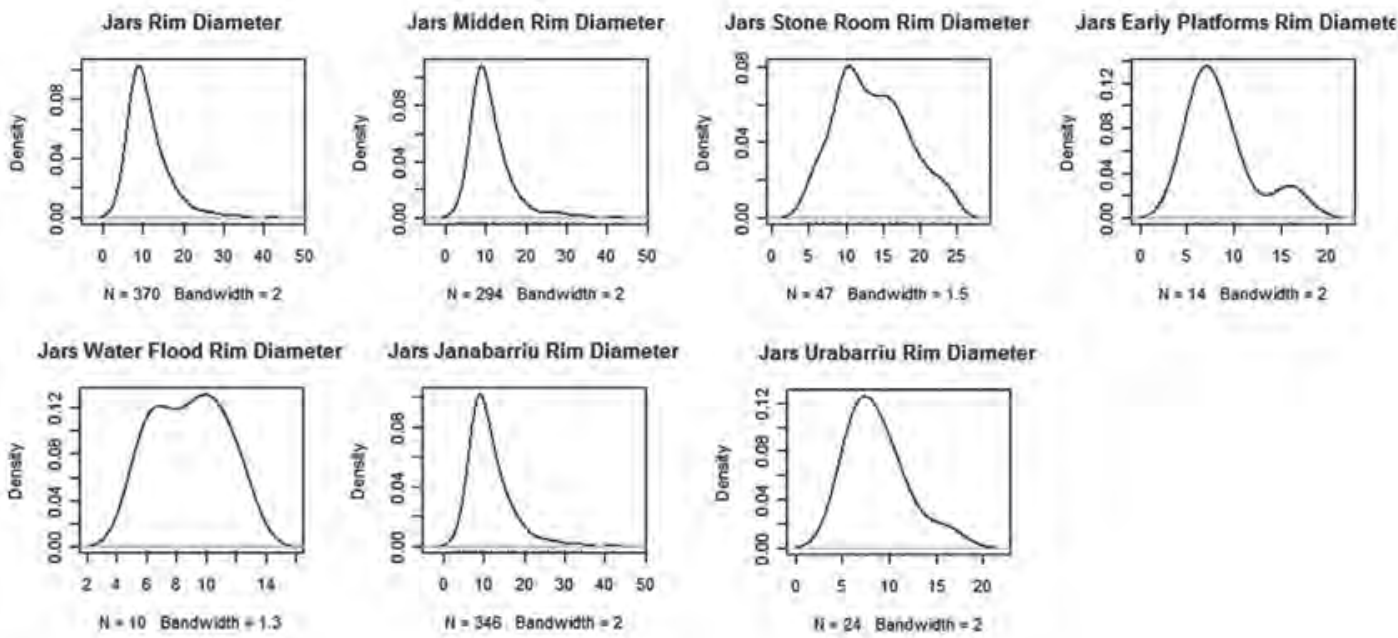

Figura 14. EDK univariados de diámetros de Jarras.

Tabla 9: Agrupaciones modales univariadas y bivariadas deJarras

\begin{tabular}{|l|l|l|l|}
\hline \multicolumn{4}{|l|}{ AGRUPACIONES MODALES UNIVARIADAS } \\
\hline KDE & Mode 1 & Mode 2 & Mode 3 \\
\hline Diámetro & $10 \mathrm{~cm}$ & Ausente & Ausente \\
\hline Grosor & $0,4 \mathrm{~cm}$ & $0,6 \mathrm{~cm}$ & 0,9 \\
\hline Basural & Mode 1 & Mode 2 & Mode 3 \\
\hline KDE & $10 \mathrm{~cm}$ & Ausente & Ausente \\
\hline Diámetro & $0,4 \mathrm{~cm}$ & $0,6 \mathrm{~cm}$ & 0,9 \\
\hline Grosor & Mode 1 & Mode 2 & Mode 3 \\
\hline Cuartos de Piedra & $15 \mathrm{~cm}$ & 23 cm \\
\hline KDE & $10 \mathrm{~cm}$ & $0,6 \mathrm{~cm}$ & 0,9 \\
\hline Diámetro & $0,4 \mathrm{~cm}$ & \multicolumn{2}{|l|}{} \\
\hline Grosor & Mode 1 & Mode 2 & Mode 3 \\
\hline Plataformas Tempranas \\
\hline KDE & $6 \mathrm{~cm}$ & $15 \mathrm{~cm}$ & Ausente \\
\hline Diámetro & $0,4 \mathrm{~cm}$ & $0,8 \mathrm{~cm}$ & Ausente \\
\hline Grosor & Mode 1 & Mode 2 & Mode 3 \\
\hline Correnteras de Agua & Ausente \\
\hline KDE & $12 \mathrm{~cm}$ & 27 \\
\hline Diámetro & $0,8 \mathrm{~cm}$ & $1,2 \mathrm{~cm}$ & Ausente \\
\hline Grosor
\end{tabular}

\begin{tabular}{|l|l|l|}
\hline \multicolumn{3}{|l|}{ AGRUPACIONES MODALES BIVARIADAS } \\
\hline Grosor & Diámetro & $\mathbf{n}$ \\
\hline 0,5392 & 9,24 & 355 \\
\hline Basural & \multicolumn{3}{|l|}{} \\
\hline Grosor & Diámetro & $\mathbf{n}$ \\
\hline 0,5392 & 8,46 & 282 \\
\hline Cuartos de Piedra \\
\hline Grosor & Diámetro & $\mathbf{n}$ \\
\hline 0,5347 & 11,65 & 36 \\
\hline Plataformas Tempranas \\
\hline Grosor & Diámetro & $\mathbf{n}$ \\
\hline 0,4112 & 6 & 5 \\
\hline 0,7136 & 7,8 & 4 \\
\hline Correntera de Agua \\
\hline Grosor & Diámetro & $\mathbf{n}$ \\
\hline 0,56 & 9,96 & 3 \\
\hline 0,59 & 6 & 2 \\
\hline 0,61 & 8,04 & 2 \\
\hline
\end{tabular}

rías son trasladadas a un gráfico EDK bivariado, un modo preponderante aparece -como se observa en la tabla 9 y en las figuras 16 y 17.

\subsubsection{Plataformas tempranas}

El tamaño de la muestra de esta unidad analítica es muy pequeño, y los resultados deben de ser tomados con mucha cautela ya que solamente se tienen 16 fragmentos. La tabla 9 muestra los resultados univariados de EDK tanto para diámetros como para grosores. Cuando estas dos categorías son tras- 

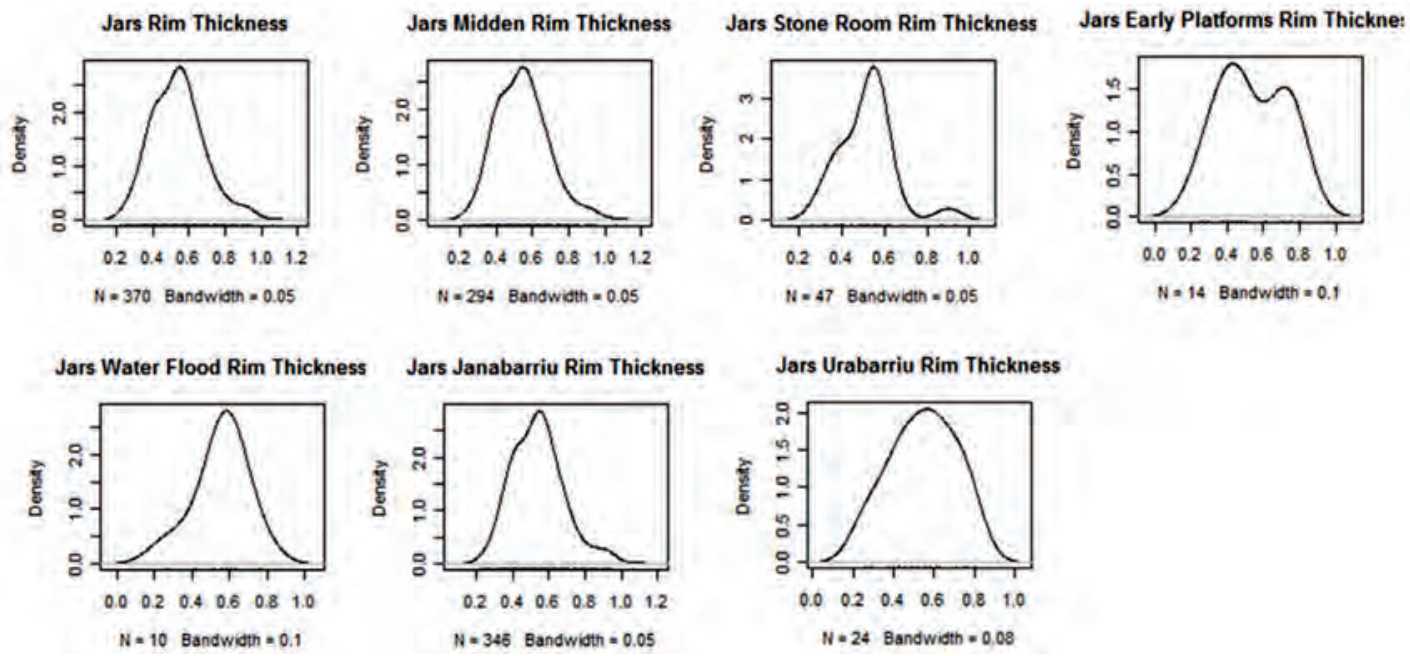

Figura 15. EDK univariados de grosores de Jarras.
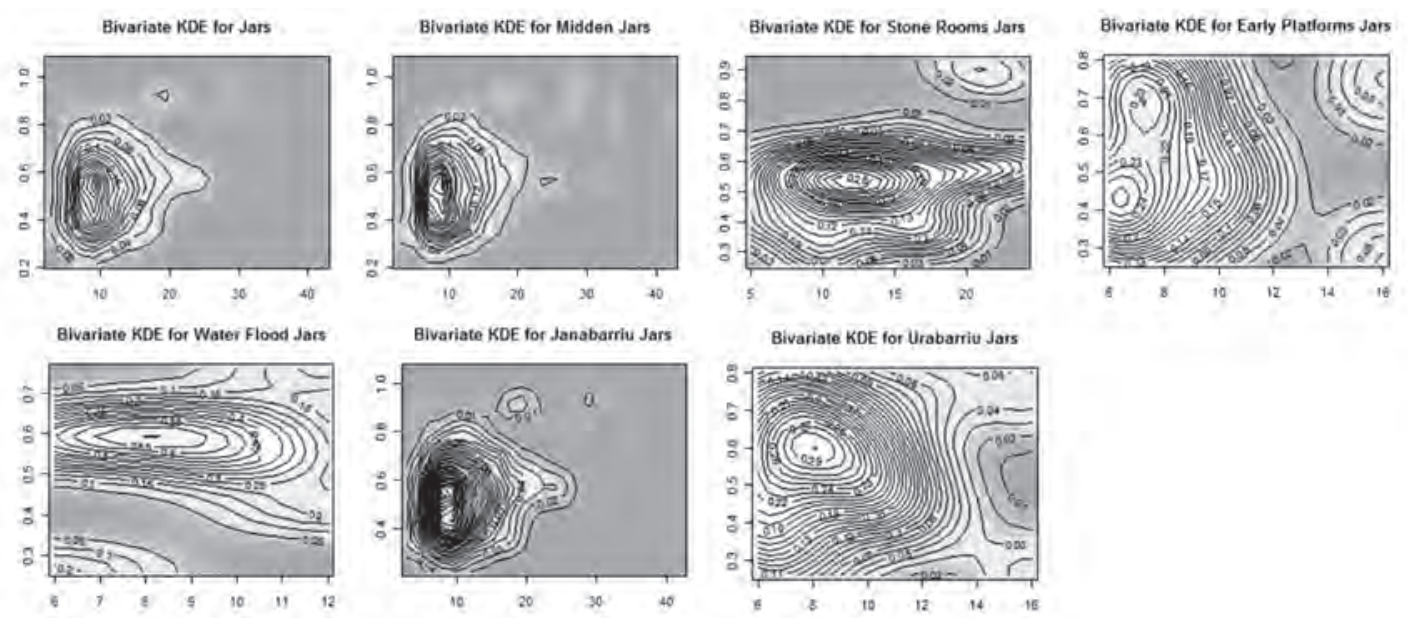

Figura 16. EDK bivariados bidimensionales de diámetros de Jarras.

ladadas a un gráfico EDK bivariado, los resultados indican la presencia de dos modos como se observa en la tabla 9 y en las figuras 16 y 17.

\subsubsection{Correntera de Agua}

Similar cautela debe de ser tomada en esta unidad analítica debido a la pequeña muestra de 14 fragmentos. La tabla 9 muestra los resultados univariados de EDK tanto para diámetros como para grosores. Las figuras 14 y 15 presentan estos modos y como se superponen. Cuando estas dos categorías son trasladadas a un gráfico EDK bivariado, los resultados indican la presencia de tres modos como se observa en la tabla 9 y en las figuras 16 y 17

\subsubsection{Plataformas Tardías}

La muestra en esta unidad analítica asciende a cinco fragmentos por lo que no hay necesidad de realizar análisis mayores. 

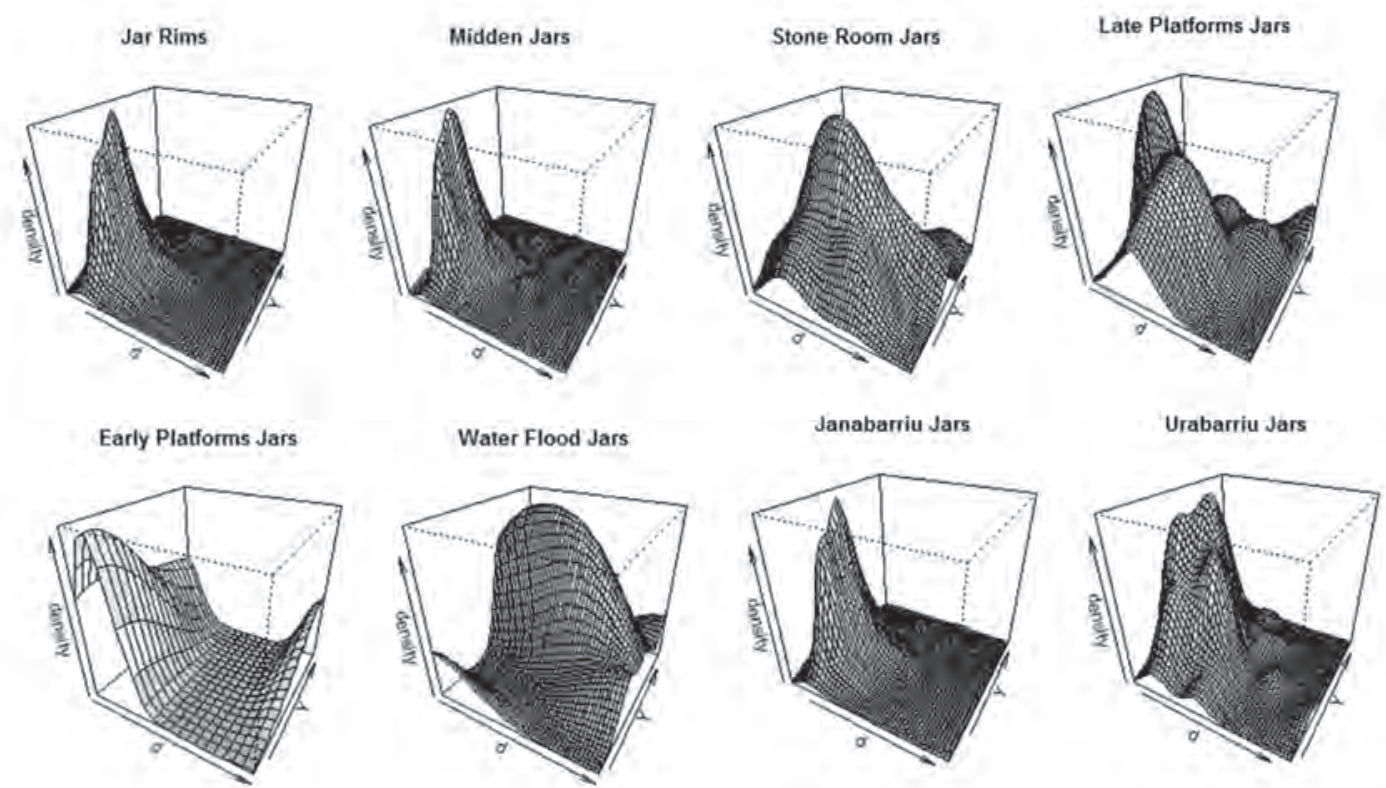

Figura 17. EDK bivariados tridimensionales de diámetros de Jarras.

En la tabla 10 puede verse un resumen de los resultados de EDK en Cuencos por unidad espacial.

\subsection{BOTELLAS}

La muestra de botellas está compuesta por 119 fragmentos, de los cuales 101 (84,9\%) es parte del Basural. Debido al reducido número de fragmentos en otras unidades espaciales, solamente las tablas serán consideradas y los patrones extraídos de ellas sin necesidad de realizar análisis EDK. Se puede argumentar que el resto de unidades espaciales no contienen una proporción significativa de botellas. La tabla 11 muestra los estimados univariados de la población total para diámetros y grosores mientras que las figuras 18 y 19 muestran como estos modos se superponen. Cuando estas dos categorías

Tabla 10: Sumario de tipos y medidas de Jarras.

\begin{tabular}{|l|c|c|c|l|}
\hline \multicolumn{1}{|c|}{ Unidad Espacial } & Muy pequeño & Pequeño & Mediano & Grande \\
\hline Basural & Ausente & $8 / 0,5$ & Ausente & Ausente \\
\hline Cuartos de Piedra & Ausente & Ausente & $12 / 0,5$ & Ausente \\
\hline Plataformas Tempranas & $6 / 0,4$ & $8 / 0,7$ & Ausente & Ausente \\
\hline Correntera de Agua & $6 / 0,6$ & $8 / 0,6$ & $10 / 0,6$ & Ausente \\
\hline Plataformas Tardías & $6 / 0,6-0,9$ & $8 / 0,3$ & Ausente & $18 / 0,9$ \\
\hline
\end{tabular}

\begin{tabular}{|l|l|l|}
\hline \multicolumn{1}{|c|}{ Unidad Espacial } & \multicolumn{1}{c|}{ Tipos } & \multicolumn{1}{c|}{ Prevalencia } \\
\hline Basural & Pequeño & Pequeño \\
\hline Cuartos de Piedra & Mediano & Mediano \\
\hline Plataformas Tempranas & Muy pequeño y pequeño & Muy Pequeño \\
\hline Correntera de Agua & Muy pequeño, pequeño y mediano & Mediano \\
\hline Plataformas Tardías & Muy pequeño, pequeño y grande & Mediano \\
\hline
\end{tabular}



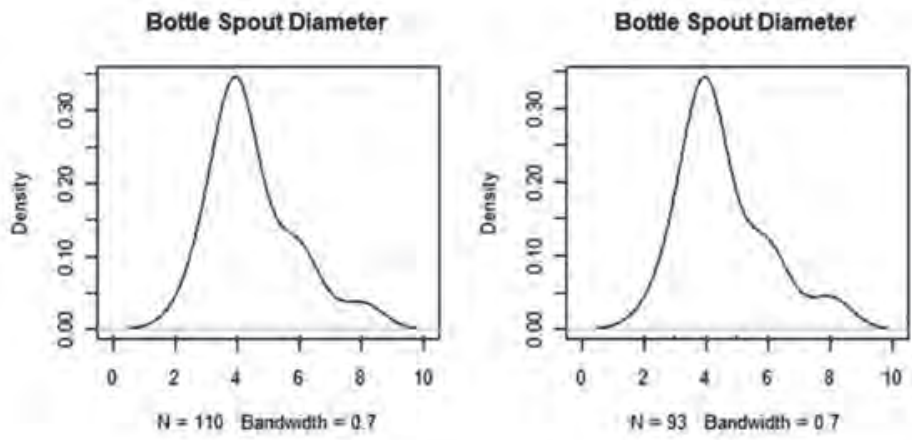

Bottle Janabarriu Spout Diameter

\section{Bottle Urabarriu Spout Diameter}
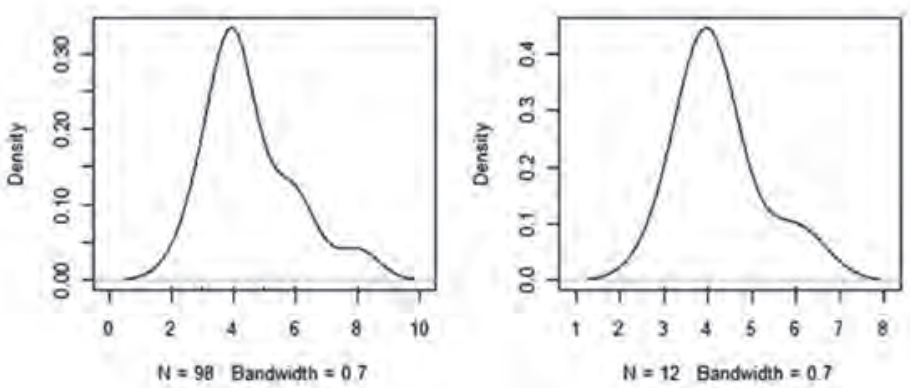

Figura 18. EDK univariados de diámetros de Botellas.
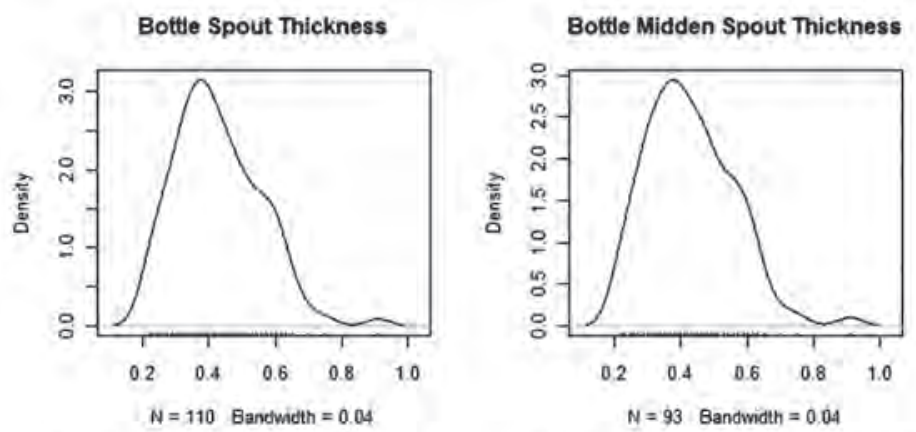

Bottle Janabarriu Spout Thickness

Bottle Urabarriu Spout Thickness
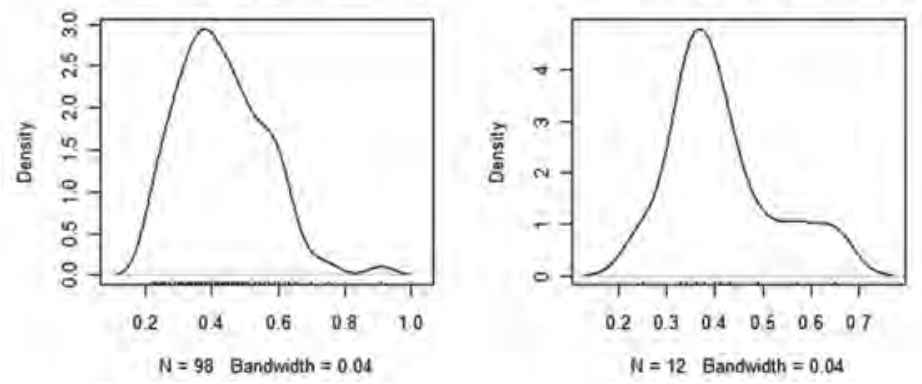

Figura 19. EDK univariados de grosores de Botellas. 
son trasladadas a un gráfico EDK bivariado, un dos modos aparecen -como se observa en la tabla 11 y en las figuras 20 y 21.

Es necesario indicar que con relación a las botellas, el diámetro del borde no indica precisamente si se trata de una botella de grandes o pequeñas dimensiones, por lo que en este artículo se lidia principalmente con la variación del borde. Los resultados obtenidos si bien no son significativos en cuanto las variaciones del tamaño de las botellas, lo son en cuento a la segregación de botellas de cuello angosto o cuello amplio, las cuales pueden estar presentes en botellas de similares dimensiones o de dimensiones generales diametralmente opuestas. El tamaño del cuello no está en función de los centímetros cúbicos de líquido que una botella puede contener.

\subsubsection{Basural}

Como se mencionó anteriormente, una muestra de 101 fragmentos ha sido cuantificada en esta unidad analítica. Dada la preponderancia de botellas del basural en la muestra general, se observa una tendencia similar a la indicada en el párrafo anterior. La tabla 11 muestra los estimados univariados de la población total para diámetros y grosores mientras que las figuras 18 y 19 muestran como estos modos se superponen. Cuando estas dos categorías son trasladadas a un gráfico EDK bivariado, un dos modos aparecen -como se observa en la tabla 11 y en las figuras 20 y 21. Dos tipos de cuellos de botella han sido identificados, pequeños $(4 / 0,4 \mathrm{~cm})$ y medianos $(6 / 0,5 \mathrm{~cm})$.

\subsubsection{Cuartos de Piedra}

Cuatro fragmentos han sido identificados en esta unidad espacial (tabla 11). Los datos provenientes de esta unidad analítica corresponden a los cuellos pequeños y medianos identificados en el Basural. Los pequeños son preponderantes si cabe la aplicación de este término a una muestra tan pequeña.

\subsubsection{Plataformas Tempranas}

Seis fragmentos han sido identificados en esta unidad espacial. Todos los casos pueden ser relacionados con el tipo pequeño reconocido en el Basural.

\subsubsection{Correntera de Agua}

Como en las unidades espaciales anteriores, el tamaño de la muestra es muy pequeño para ser sometido a análisis EDK. De lo observado en la tabla 12, se tiene la presencia de botellas con cuello pequeño y mediano.

\subsubsection{Plataformas Tardías}

Solamente un fragmento de $5 / 0,24 \mathrm{~cm}$ se ha identificado en la muestra proveniente de esta unidad analítica, lo que corresponde al tipo mediano reconocido en el basural.

Un sumario con respecto a las botellas es observado en la tabla 12. Nuevamente es necesario precisar que los valores que provienen de CP, PT, PTA y CA deben de ser tomados con extrema cautela.

\subsection{VASOS}

La muestra de vasos en esta unidad analítica está compuesta tan solo por 40 fragmentos, de los cuales 37 (92,5\%) provienen del Basural. Dado el número reducido de de fragmentos de vasos en otras unidades espaciales se seguirá el mismo procedimiento utilizado para las botellas. La tabla 13 muestra los estimados univariados de la población total para diámetros y grosores mientras que la figura 22 
Christian Mesía / Uso de estimados de densidad Kernel en la investigación de grupos cerámicos...

Tabla 11: Agrupaciones modales univariadas y bivariadas de Botellas

\begin{tabular}{|l|l|l|l|}
\hline \multicolumn{5}{|l|}{ AGRUPACIONES MODALES UNIVARIADAS } \\
\hline KDE & Mode 1 & Mode 2 & Mode 3 \\
\hline Diámetro & $4 \mathrm{~cm}$ & $6 \mathrm{~cm}$ & $8 \mathrm{~cm}$ \\
\hline Grosor & $0,4 \mathrm{~cm}$ & $0,6 \mathrm{~cm}$ & $0,9 \mathrm{~cm}$ \\
\hline Basural & Mode 1 & Mode 2 & Mode 3 \\
\hline KDE & $6 \mathrm{~cm}$ & $8 \mathrm{~cm}$ \\
\hline Diámetro & $4 \mathrm{~cm}$ & $0,9 \mathrm{~cm}$ \\
\hline Grosor & $0,4 \mathrm{~cm}$ & $0,6 \mathrm{~cm}$ & Mode 3 \\
\hline Cuartos de Piedra & Mode 2 & $23 \mathrm{~cm}$ \\
\hline KDE & Mode 1 & $15 \mathrm{~cm}$ & $0,9 \mathrm{~cm}$ \\
\hline Diámetro & $10 \mathrm{~cm}$ & $0,6 \mathrm{~cm}$ & Mode 3 \\
\hline Grosor & $0,4 \mathrm{~cm}$ & Mode 2 & Ausente \\
\hline Plataformas Tempranas & $15 \mathrm{~cm}$ & Ausente \\
\hline KDE & Mode 1 & $0,8 \mathrm{~cm}$ & Mode 3 \\
\hline Diámetro & $6 \mathrm{~cm}$ & & Ausente \\
\hline Grosor & $0,4 \mathrm{~cm}$ & Mode 2 & Ausente \\
\hline Correnteras de Agua & Mode 1 & $27 \mathrm{~cm}$ & \\
\hline KDE & $12 \mathrm{~cm}$ & $1,2 \mathrm{~cm}$ & \\
\hline Diámetro & $0,8 \mathrm{~cm}$ & \\
\hline Grosor & \multicolumn{5}{|l}{} \\
\hline
\end{tabular}

\begin{tabular}{|c|c|c|}
\hline \multicolumn{3}{|c|}{ AGRUPACIONES MODALES BIVARIADAS } \\
\hline Grosor & Diámetro & $n$ \\
\hline 0.5392 & 9.24 & 355 \\
\hline \multicolumn{3}{|l|}{ Basural } \\
\hline Grosor & Diámetro & $\mathbf{n}$ \\
\hline 0,5392 & 8,46 & 282 \\
\hline \multicolumn{3}{|c|}{ Cuartos de Piedra } \\
\hline Grosor & Diámetro & $\mathrm{n}$ \\
\hline 6 & 0,6 & 1 \\
\hline 3 & 0,42 & 13 \\
\hline 3 & 0,36 & 13 \\
\hline 3 & 0,51 & 13 \\
\hline \multicolumn{3}{|c|}{ Plataformas Tempranas } \\
\hline Grosor & Diámetro & $\mathrm{n}$ \\
\hline 4 & 0,49 & 1 \\
\hline 4 & 0,65 & 1 \\
\hline 4 & 0,57 & 1 \\
\hline 4 & 0,33 & 1 \\
\hline 4 & 0,25 & 1 \\
\hline 4 & 0,4 & 1 \\
\hline \multicolumn{3}{|c|}{ Correntera de Agua } \\
\hline Grosor & Diámetro & $\mathbf{n}$ \\
\hline 4 & 0,43 & 1 \\
\hline 4 & 0,38 & 1 \\
\hline 4 & 0,41 & 1 \\
\hline 3 & 0,34 & 1 \\
\hline 6 & 0,36 & 1 \\
\hline 6 & 0,34 & 1 \\
\hline
\end{tabular}
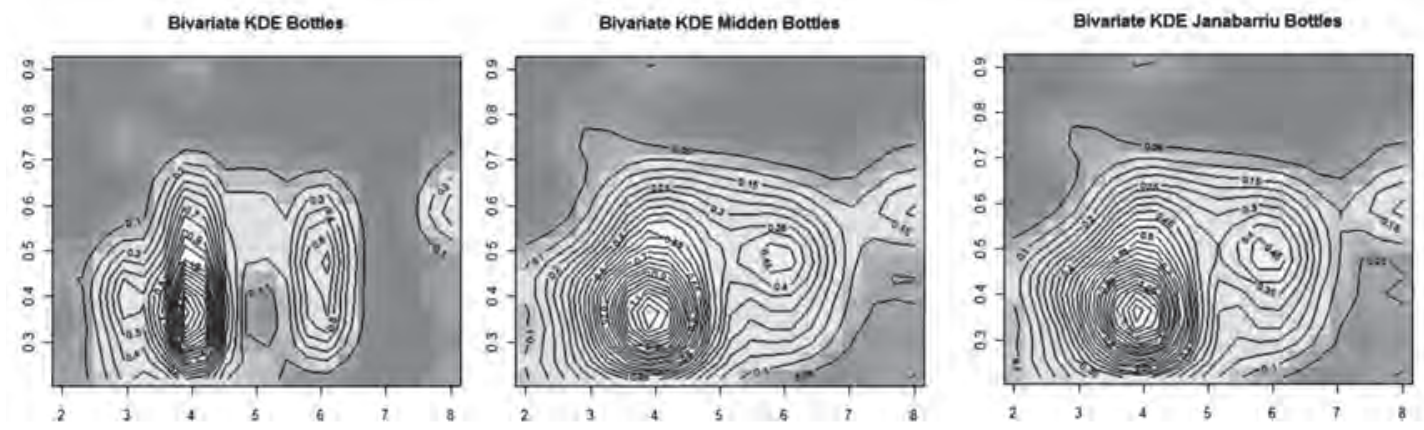

Figura 20. EDK bivariados bidimensionales de diámetros de Botellas

muestra como estos modos se superponen. Cuando estas dos categorías son trasladadas a un gráfico EDK bivariado, cinco modos aparecen -como se observa en la tabla 13 y en las figuras 23 y 24 . Debe de indicarse que el modo pequeño es increíblemente pequeño y podría tratarse de vasos miniatura.

\subsubsection{Basural}

La muestra de vasos identificada en el Basural corresponde a 37 fragmentos. Se replican los valores identificados en la muestra total de vasos. 
Bottle Spouts

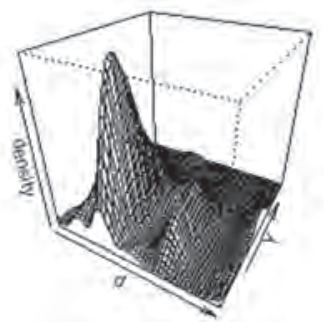

Water Flood Bottles

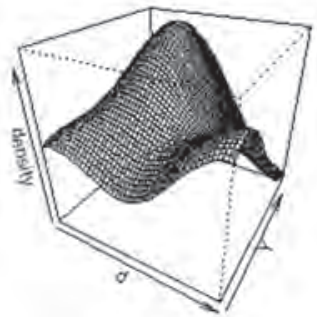

Midden Bottles

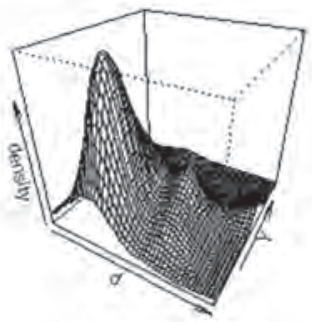

Janabarriu Bottles

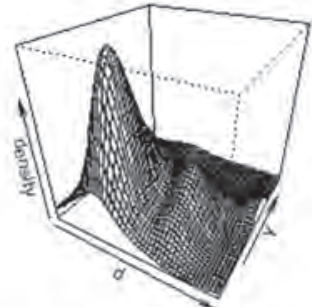

Stone Room Bottles

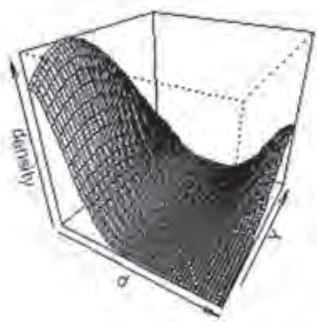

Urabarriu Bottles

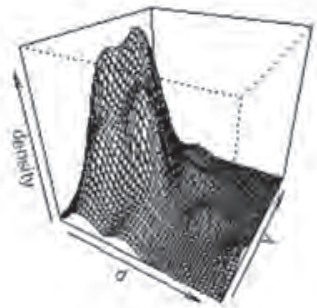

Early Platforms Bottles

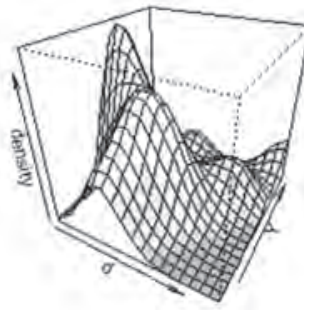

Figura 21. EDK bivariados tridimensionales de diámetros de Botellas.

Tabla 12: Sumario de tipos y medidas de Jarras.

\begin{tabular}{|l|l|l|}
\hline Unidad Espacial & Pequeño & Mediano \\
\hline Basural & $4 / 0,4$ & $6 / 0,5$ \\
\hline Cuartos de Piedra & $4 / 0,4-0,5$ & $6 / 0,6$ \\
\hline Plataformas Tempranas & $4 / 0.3-0.7$ & Ausente \\
\hline Correntera de Agua & $3-4 / 0,3-0,4$ & $6 / 0,3-0,4$ \\
\hline Plataformas Tardías & Ausente & $5 / 0,2$ \\
\hline
\end{tabular}

\begin{tabular}{|l|l|l|}
\hline Unidad Espacial & Tipo & Prevalencia \\
\hline Basural & Pequeño, mediano & Pequeño \\
\hline Cuartos de Piedra & Pequeño, mediano & Pequeño \\
\hline Plataformas Tempranas & Pequeño & Pequeño \\
\hline Correntera de Agua & Pequeño, mediano & Pequeño \\
\hline Plataformas Tardías & Mediano & Mediano \\
\hline
\end{tabular}
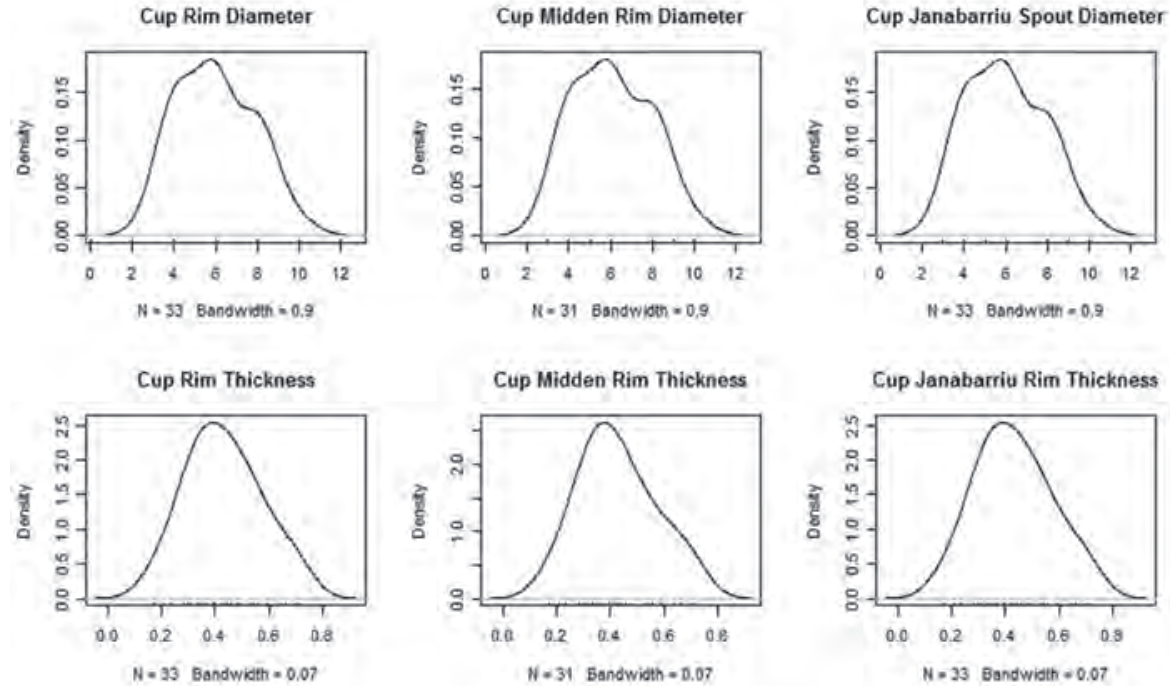

Cup Janabarriu Rim Thickness

Figura 22. EDK univariados de diámetros y grosores de Vasos. 

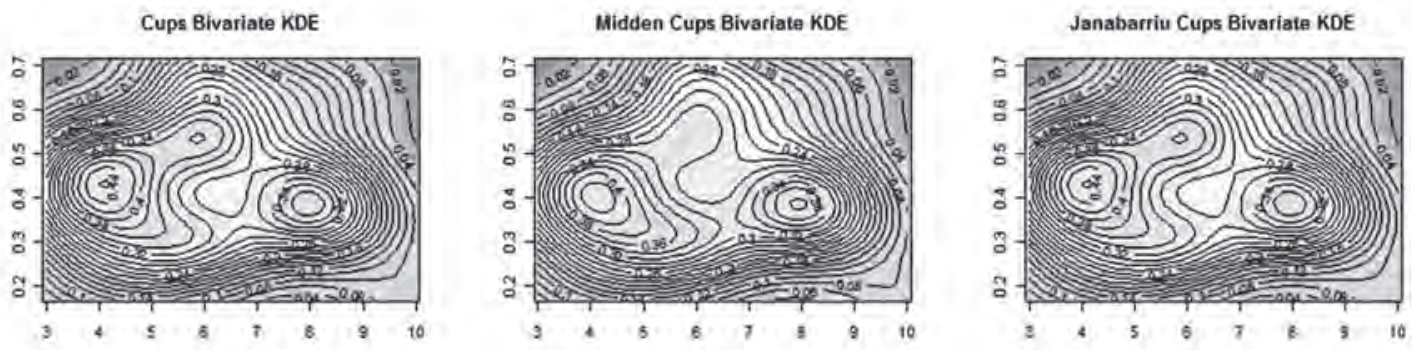

Figura 23. EDK bivariados bidimensionales de diámetros de Vasos.
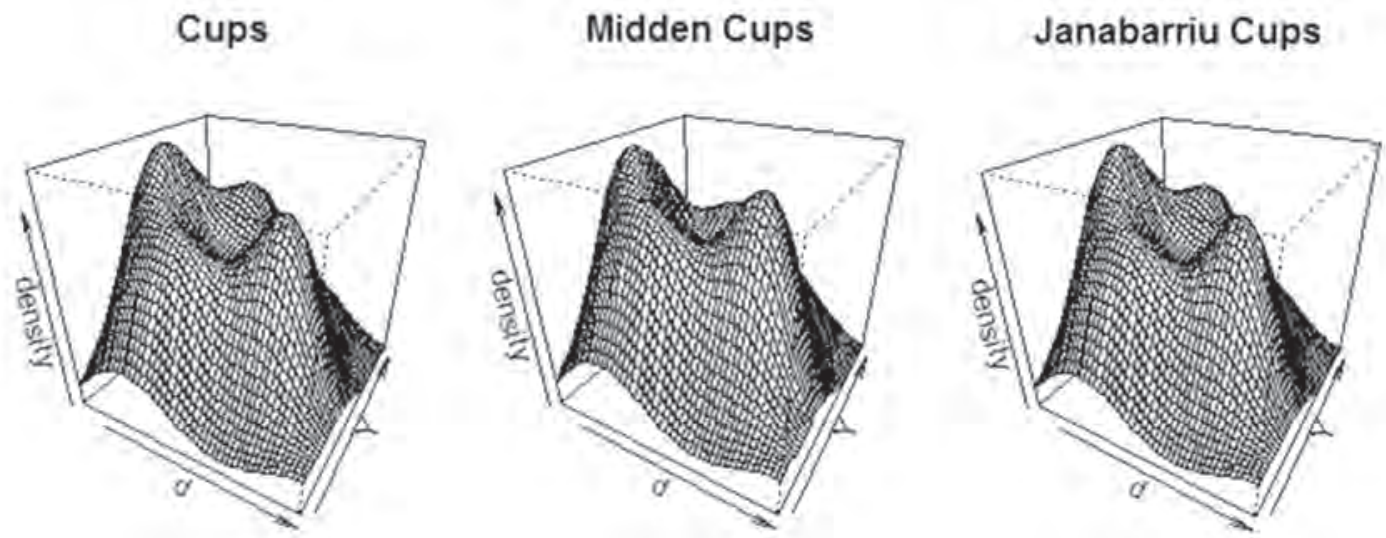

Figura 24. EDK bivariados bidimensionales de diámetros de Vasos.

Tabla 13: Agrupaciones modales univariadas y bivariadas de Vasos.

\begin{tabular}{|l|l|l|l|}
\hline \multicolumn{4}{|l|}{ AGRUPACIONES MODALES UNIVARIADAS } \\
\hline KDE & Mode 1 & Mode 2 & Mode 3 \\
\hline Diámetro & $4 \mathrm{~cm}$ & $6 \mathrm{~cm}$ & $8 \mathrm{~cm}$ \\
\hline Grosor & $0,4 \mathrm{~cm}$ & Ausente & Ausente \\
\hline Basural \\
\hline KDE & Mode 1 & Mode 2 & Mode 3 \\
\hline Diámetro & $4 \mathrm{~cm}$ & $6 \mathrm{~cm}$ & $8 \mathrm{~cm}$ \\
\hline Grosor & $0,4 \mathrm{~cm}$ & Ausente & Ausente \\
\hline
\end{tabular}

\begin{tabular}{|l|l|l|}
\hline \multicolumn{4}{|l|}{ AGRUPACIONES MODALES BIVARIADAS } \\
\hline Grosor & Diámetro & $\mathbf{n}$ \\
\hline 0,2996 & 5,94 & 5 \\
\hline 0,3968 & 8,04 & 7 \\
\hline 0,44 & 3,98 & 10 \\
\hline 0,5372 & 5,94 & 7 \\
\hline 0,6236 & 8,04 & 2 \\
\hline Basural & \multicolumn{2}{|l|}{} \\
\hline Grosor & Diámetro & $\mathbf{n}$ \\
\hline 0,2996 & 5,52 & 5 \\
\hline 0,386 & 8,04 & 7 \\
\hline 0,4184 & 3,84 & 10 \\
\hline 0,548 & 5,94 & 3 \\
\hline 0,6236 & 8,04 & 2 \\
\hline
\end{tabular}




\subsubsection{Cuartos de Piedra}

Solamente se ha identificado un fragmento, cuyo valor es de 4/0,43 cm. Se relaciona con la categoría de vasos pequeños identificada en el Basural.

\subsubsection{Plataformas Tempranas}

No se recuperaron vasos en esta unidad espacial.

\subsubsection{Correntera de Agua}

Solamente se ha identificado un fragmento, cuyo valor es de 4/0,31 cm. Se relaciona con la categoría de vasos pequeños identificada en el Basural.

\subsubsection{Plataformas Tardías}

Solamente se ha identificado un fragmento, cuyo valor es de $6 / 0,53 \mathrm{~cm}$. Se relaciona con la categoría de vasos medianos y gruesos identificada en el Basural.

Con las reservas ya indicadas respecto al tamaño de la muestra de vasos, la misma puede ser organizada tal cual se observa en la tabla 14.

Tabla 14: Sumario de tipos y medidas de Vasos.

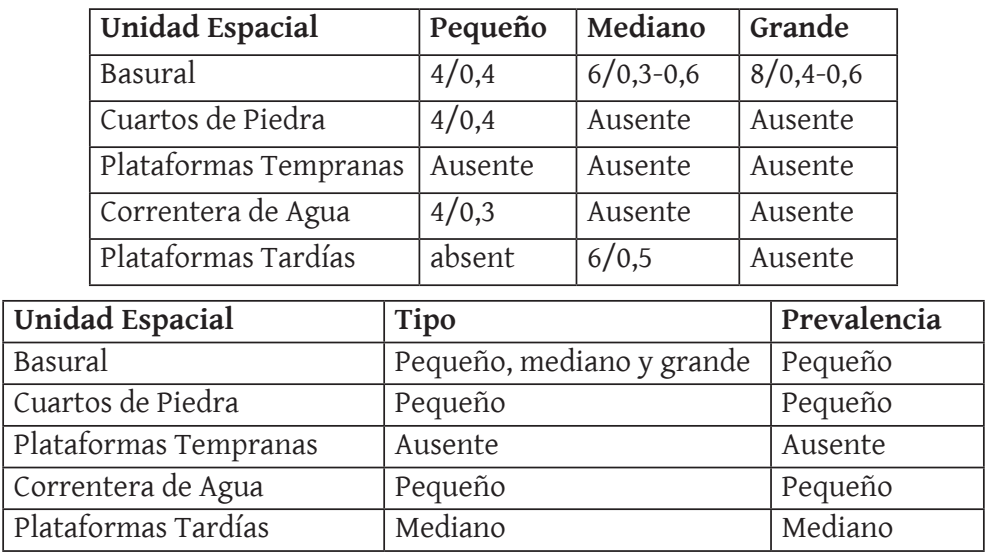

\subsection{PLATOS}

La población de platos está compuesta por 57 fragmentos, de los cuales $46(80,1 \%)$ pertenece al Basural. Dado el número reducido de de fragmentos de vasos en otras unidades espaciales se seguirá el mismo procedimiento utilizado para las botellas y vasos. Esta baja densidad invita a pensar que los platos no fueron realmente parte importante de la muestra cerámica recuperada en el sector Wacheqsa. La tabla 15 muestra los estimados univariados de la población total para diámetros y grosores mientras que la figura 25 muestra como estos modos se superponen. Cuando estas dos categorías son trasladadas a un gráfico EDK bivariado, dos modos aparecen como se observa en la tabla 15 y en las figuras 26 y 27.

\subsubsection{Basural}

Como se mencionó en el párrafo anterior, la muesta de platos en el Basural es de 46 fragmentos, replicándose los valores identificados en la muestra general. Los datos referentes a las otras unidades espaciales pueden ser observados en la tabla 16. 
Christian Mesía / Uso de estimados de densidad Kernel en la investigación de grupos cerámicos...

Tabla 15: Agrupaciones modales univariadas y bivariadas de Platos.

\begin{tabular}{|l|l|l|l|}
\hline \multicolumn{4}{|l|}{ AGRUPACIONES MODALES UNIVARIADAS } \\
\hline KDE & Mode $\mathbf{1}$ & Mode $\mathbf{2}$ & Mode 3 \\
\hline Diámetro & $15 \mathrm{~cm}$ & $25 \mathrm{~cm}$ & Ausente \\
\hline Grosor & $0,6 \mathrm{~cm}$ & $0,8 \mathrm{~cm}$ & Ausente \\
\hline Basural \\
\hline KDE & Mode 1 & Mode 2 & Mode 3 \\
\hline Diámetro & $15 \mathrm{~cm}$ & $25 \mathrm{~cm}$ & Ausente \\
\hline Grosor & $0,6 \mathrm{~cm}$ & $0,8 \mathrm{~cm}$ & Ausente \\
\hline
\end{tabular}

Tabla 16: Sumario de tipos y medidas de Vasos

\begin{tabular}{|l|l|l|l|}
\hline Unidad Espacial & Pequeño & Mediano & Grande \\
\hline Basural & absent & absent & $22 / 0,9$ \\
\hline Cuartos de Piedra & $10 / 0,4$ & $18 / 0,6$ & $22-26 / 0,9$ \\
\hline Plataformas Tempranas & $12 / 0,6$ & Ausente & $24 / 0,7$ \\
\hline Correntera de Agua & $14 / 0,9$ & Ausente & $26 / 0,51$ \\
\hline Plataformas Tardías & Ausente & Ausente & Ausente \\
\hline Unidad Espacial & Tipo & Prevalencia \\
\hline Basural & Grande & Grande \\
\hline Cuartos de Piedra & $\begin{array}{l}\text { Pequeño, mediano } \\
\text { ygrande }\end{array}$ & Grande \\
\hline Plataformas Tempranas & Pequeño y grande & $\begin{array}{l}\text { Pequeño y } \\
\text { grande }\end{array}$ \\
\hline Correntera de Agua & Pequeño y grande & $\begin{array}{l}\text { Pequeño y } \\
\text { grande }\end{array}$ \\
\hline Plataformas Tardías & Ausente & Ausente \\
\hline
\end{tabular}

\begin{tabular}{|l|l|l|}
\hline \multicolumn{3}{|l|}{ AGRUPACIONES MODALES BIVARIADAS } \\
\hline Grosor & Diámetro & $\mathbf{n}$ \\
\hline 0,5724 & 12,4 & 6 \\
\hline 0,615 & 22 & 40 \\
\hline Basural & \multicolumn{3}{|l|}{ Diámetro } & $\mathbf{n}$ \\
\hline Grosor & D & 38 \\
\hline 0,615 & 22 & 3 \\
\hline 0,9132 & 22 & $\mathbf{n}$ \\
\hline Cuartos de Piedra \\
\hline Grosor & Diámetro & $\mathbf{n}$ \\
\hline 22 & 0,82 & 1 \\
\hline 26 & 0,63 & 1 \\
\hline 24 & 0,82 & 1 \\
\hline 18 & 0,63 & 1 \\
\hline 10 & 0,4 & 1 \\
\hline 10 & 0,27 & 1 \\
\hline Plataformas Tempranas \\
\hline Grosor & Diámetro & $\mathbf{n}$ \\
\hline 12 & 0,56 & 1 \\
\hline 24 & 0,69 & 1 \\
\hline Correntera de Agua \\
\hline Grosor & Diámetro & $\mathbf{n}$ \\
\hline 26 & 0,51 & 1 \\
\hline 14 & 0,92 & 1 \\
\hline \multicolumn{4}{|c|}{} \\
\hline
\end{tabular}

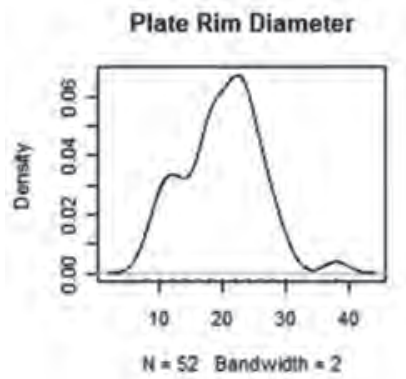

Plate Rim Thickness

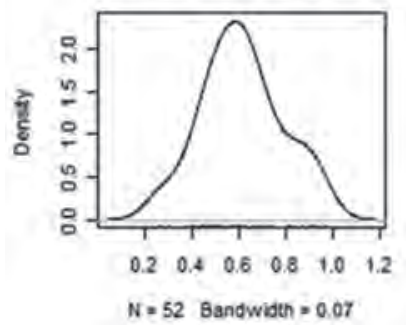

Plate Midden Rim Diameter

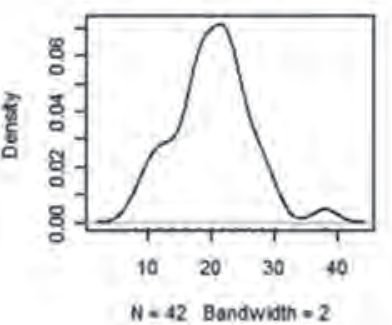

Plate Midden Rim Thickness

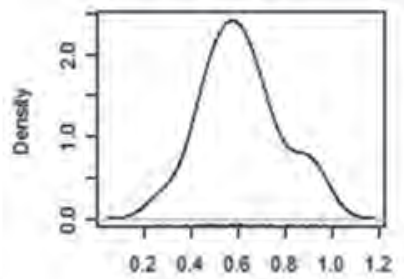

$N=42$ Bandwieth $=0.07$
Plate Janabarriu Rim Diameter

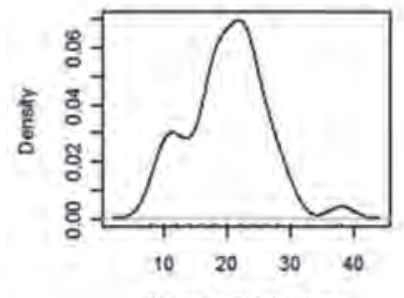

$N=48$ Bandwidth $=2$

Plate Janabarriu Rim Thickness

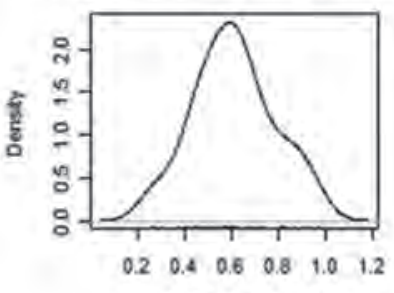

$N=48$ Bandwith $=0.07$

Figura 25. EDK univariados de diámetros y grosores de Platos. 

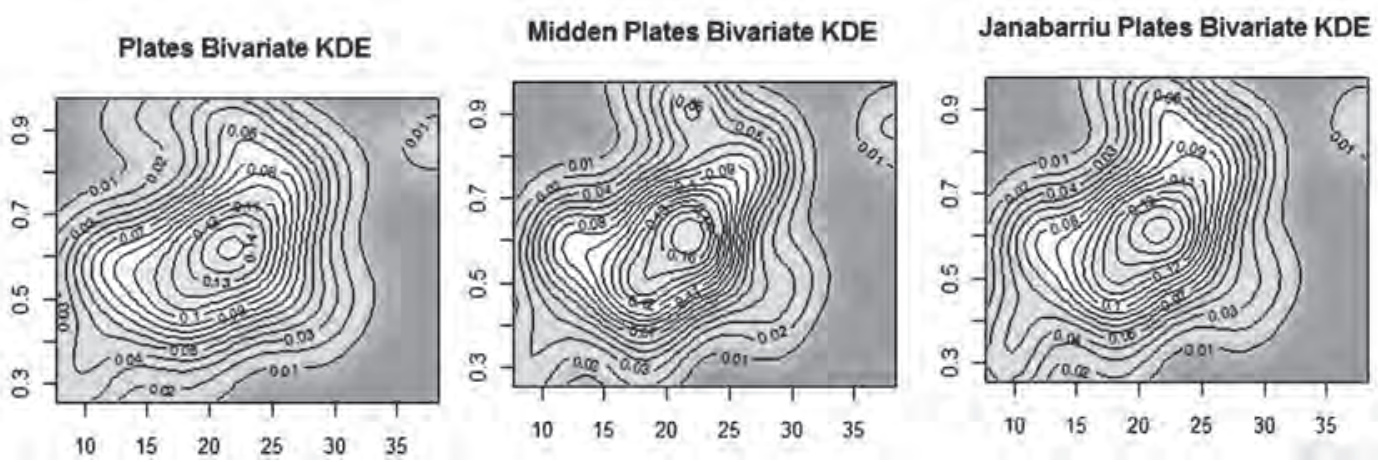

Figura 26. EDK bivariados bidimensionales de diámetros de Platos.

Plates

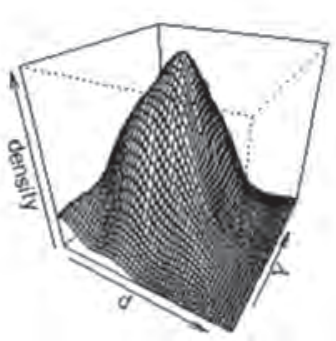

Midden Plates

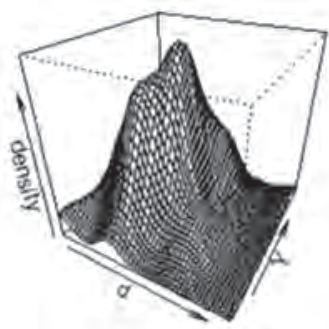

Janabarriu Plates

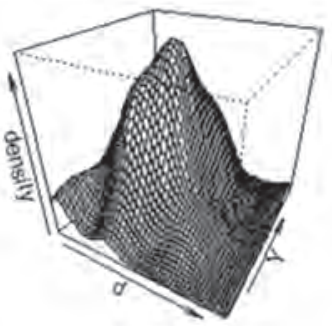

Figura 27. EDK bivariados tridimensionales de diámetros de Platos.

Asimismo la tabla 16 muestra una sinopsis de la distribución de platos por unidad espacial. Nuevamente es muy importante recalcar la cautela con la cual los valores provenientes de CP, PT, PTA y CA deberán ser tomados.

Existe una clara variación del grupo cerámico muestreado, la cual refleja diferencias en tipos prevalentes entre unidades espaciales, tal cual se observa en la tabla 17. La naturaleza de estas funciones será discutida en un siguiente artículo pero para aquellos interesados, sugiero revisar la tesis doctoral del autor (Mesia 2007).

\section{Discusión}

Ciertas consideraciones necesitan ser comentadas al observar los resultados de la sección precedente. Los resultados provenientes de unidades espaciales con densidades altas tienen un grado de certeza indudablemente mayor que aquellos que provienen de unidades espaciales con densidades bajas, encerrando la posibilidad de crear artefactos estadísticos en cada modo identificado, especialmente cuando existen outliers en la muestra. Esto se aplica también a las tipos cerámicos con bajas densidades al interior de las unidades espaciales. Esto se observa por ejemplo en la muestra de botellas, vasos y platos que pertenecen a las unidades espaciales de la fase Urabarriu. Es necesario realizar un ejercicio de cautela y no interpretar los resultados de estos tipos cerámicos en estas unidades espaciales como modos infalibles sino más bien como educadas presunciones hasta contar con una muestra mayor. Para los análisis bivariados e incluso univariados, "el problema es conocer que es lo que constituye una muestra adecuada ya que esto depende de la estructura subyacente de los datos" (Baxter et al. 2000). En el ejemplo de análisis bivariados de 62 especímenes que Baxter et al. dan en el mismo artículo (Baxter et al. 2000), este número es a duras penas satisfactorio, sin embargo por fines 
didácticos y siguiendo las recomendaciones de Baxter et al, se eligió la muestra de 30 como mínimo para realizar los análisis que motivan el presente artículo, ya que la prevalencia de modos fabricados es muy alta en una muestra menor de 30.

En referencia a la relación diámetro/grosor, la primera medida resiste satisfactoriamente los resultados de análisis bivariados en relación a los univariados por lo que es más robusto en el examen de dimensiones de vasijas que la medida de grosor. Los modos detectados en EDK univariados han resistido en su gran mayoría la prueba de los EDK bivariados.

Otro elemento que vale la pena recalcar es la necesidad de razonar en términos tridimensionales la relación entre dos categorías que conforman un análisis bivariado ya que los gráficos en dos dimensiones no han identificado correctamente los modos estadísticos detectados en gráficos tridimensionales para el caso de las ollas sin cuello del Basural y los Cuartos de Piedra. Un elemento notable de control de los análisis EDK realizados en $\mathrm{R}$ es la tabla de agrupamiento que se genera a partir de rutinas EDK realizadas en JMP. El uso de rutinas EDK en ambos paquetes estadísticos genera una mayor robustez a los resultados, teniendo siempre en cuenta que el tamaño de la muestra debe de ser el adecuado.

En resumen se puede indicar que las rutinas EDK, fundamentalmente las bivariadas tridimensionales se constituyen en poderosas herramientas analíticas para el examen de relaciones muy superiores a los histogramas, lo cual expresa concordancia con las salvedades establecidas por Baxter (Baxter et al. 1997), Cleveland (Cleveland 1993), Shennan (Shennan 2006) Su potencial en arqueología no ha sido plenamente explotado por lo que es de esperar que el presente artículo genere un mayor uso del mismo entre los profesionales de la arqueología interesados en herramientas analíticas de investigación.

\section{Agradecimientos}

Los trabajos de excavación en Chavín de Huántar fueron realizados con apoyo del Departamento de Ciencias Antropológicas de la Universidad de Stanford, el Centro de Estudios Latinoamericanos de la Universidad de Stanford, la Facultad de Humanidades de la Universidad de Stanford y la National Geographic. A Ian Robertson quien fue instrumental en el desarrollo del presente análisis estadístico al introducirme a la programación estadística y al uso de $\mathrm{R}$, además de realizar valiosos comentarios al mismo. A John Rick por igualmente contribuir al desarrollo de la presente investigación y por su camaradería reflejada a lo largo de los años tanto en Stanford como en Chavín de Huantar. Igualmente a mis colegas y amigos John Wolf y Daniel Contreras con quienes he compartido discusiones extensas sobre los resultados de estos análisis. Desde luego los errores incluidos en el presente trabajo son de exclusiva responsabilidad mía. 


\section{BiBLIOGRAFÍA}

BAXTER, M.

2003 Statistics in Archaeology. New York: First ed. Arnold Publishers.

BAXTER, M. J. y C. C. BEARDAH

1996 "Beyond the histogram improved approaches to simple data display in archaeology using kernel density estimates". Archeologia e Calcolatori VII: 397-408.

BAXTER, M. J., C. C. BEARDAH y S. WESTWOOD

2000 "Sample size and related issues in the Analysis of Lead Isotope Data". Journal of Archaeological Science 27: 973-980.

BAXTER, M. J., C. C. BEARDAH y R. V. S. WRIGHT

1997 "Some Archaeological Applications of Kernel Density Estimates". Journal of Archaeological Science 24(4): 347-354.

BLITZ, J. H.

1993 "Big Pots for Big Shots: Feasting and Storage in a Mississippian Community". American Antiquity 58(1): 80.

BOWMAN, A. y P. FOSTER

1992 "Adaptive smoothing and density based tests of multivariate normality". Journal of the American Statistics Association (88): 529-537.

BURGER, R.

1984 The Prehistoric Occupation at Chavín de Huántar, Perú. University of Calfornia Publications, Anthropology 14. University of Berkeley, Berkeley.

1993 “The Chavin Horizon: Stylistic Chimera or Socioeconomic Metamorphosis?". En: Latin American Horizons, edited by D. S. Rice, pp. 41:82. Dumbarton Oaks, Washington.

BURGER, R. L.

1992 Chavin and the origins of Andean civilization. Londres: Thames and Hudson.

1998 Excavaciones en Chavin de Huantar. Lima: Pontificia Universidad Catolica del Perú.

CLEVELAND, W. S.

1993 Visualising data. New Jersey: Hobart Press.

DRENNAN, R.

1996 Statistics for Archaeologists, A Common Sense Approach. New York: First ed. Plenum Press.

KEMBEL, S. y J. RICK

2004 "Building Authority at Chavín de Huántar: Models of Social Development in the Initial Period and Early Horizon”. En: Andean Archaeology, editado por H. Silverman. pp. 51-76. Oxford: Blackwell Publishing.

LONGACRE, W.

1999 “Standardization and Specialization: What's the Link?". En: Pottery and People, A Dynamic Interaction, editado por J. Skibo and G. Feinman, pp. 44-58. Foundations of Archaeological Inquiry. University of Utah Press, Utah.

MESIA, C.

2007 Intrasite Spatial Organization at Chavín de Huántar during the Andean Formative: Three Dimensional Modeling, Stratigraphy and Ceramics, Stanford University.

MILLS, B.

1999 "Ceramics and Social Contexts of Food Comsumption". En: Pottery and People, A Dynamic Interaction, editado por J. Skibo y G. Feinman, pp. 99-114. Utah: Foundations of Archaeological Inquiry. University of Utah Press.

1988 "Review of Quatitative Research in Archaeology". Antiquty 62: 597-598. 


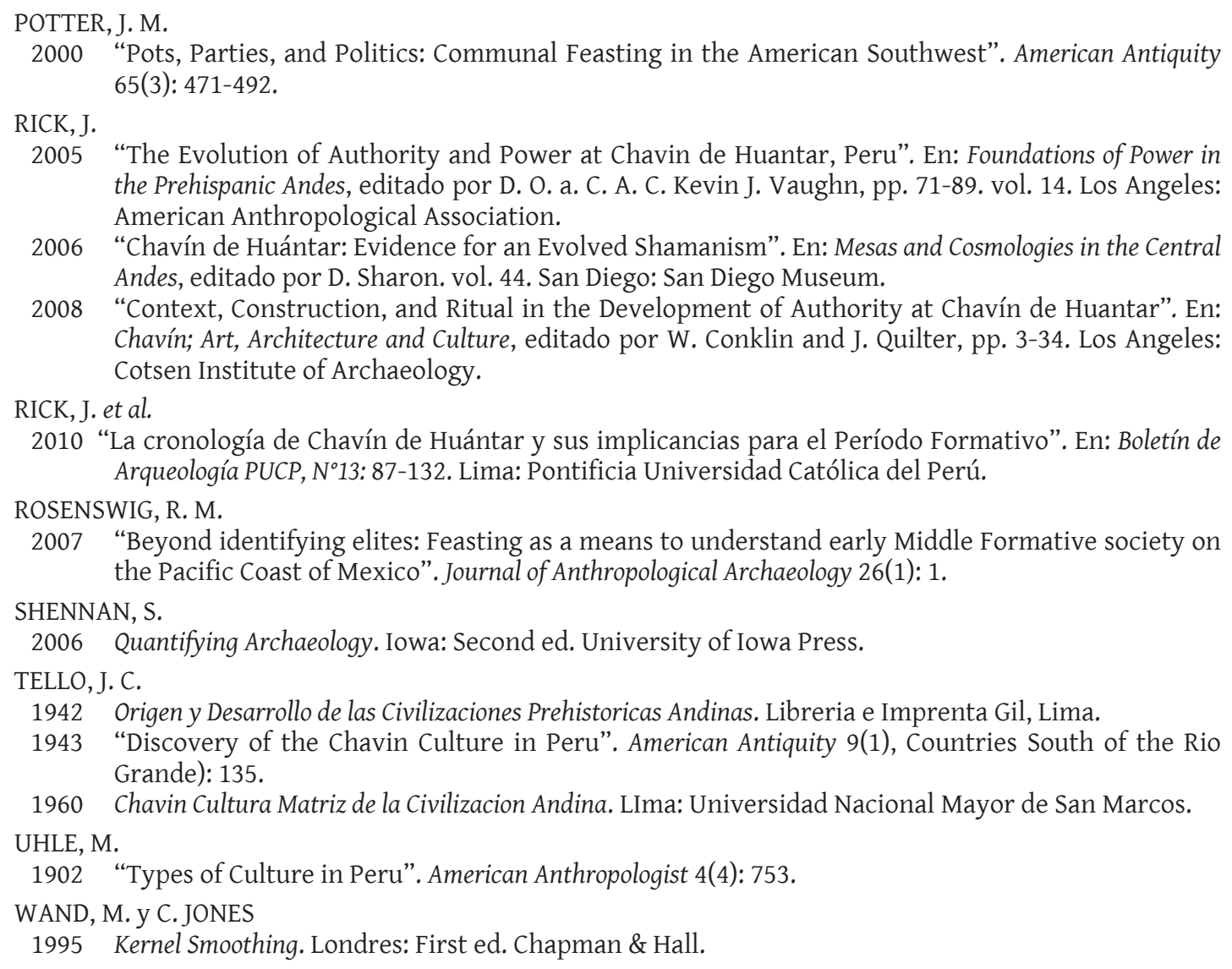




\section{APÉNDICES}

I. Código de programación R para rutinas EDK univariadas http://addictedtor.free.fr/graphiques/graphcode.php?graph=103

require(hdrcde)

data(faithful)

hdr.den(faithful\$eruptions)

rug(faithful\$eruptions, ticksize= 0.01)

II. Código de programación R para rutinas EDK bivariadas 2D y 3D http://addictedtor.free.fr/graphiques/graphcode.php?graph=103

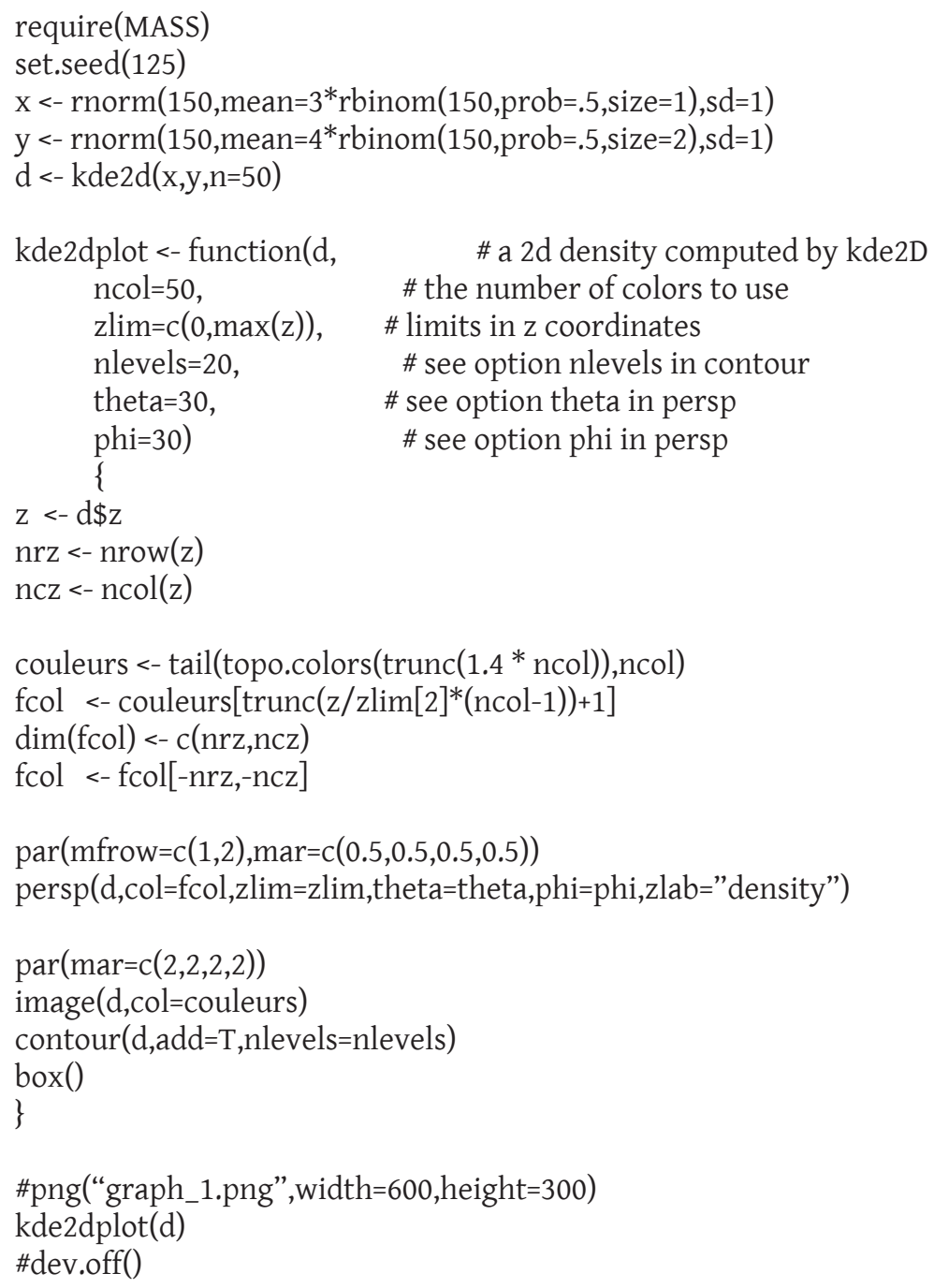
kde2dplot(d) \#dev.off() 